\title{
Sugli spazii che ammettono un gruppo continuo di movimenti.
}

\author{
(Di Guido Fubini, a Pisa.)
}

MEMORIA I.

Questo lavoro e un altro che mi riservo di pubblicare fra breve trattano della teoria generale degli spazii che ammettono un gruppo continuo di movimenti, delle proprietà di questi gruppi, dei loro sottogruppi finiti discontinui, e della determinazione degli spazii a quattro dimensioni che ammettono un tale gruppo di movimenti. I metodi di cui il prof. Bianchi si servl $\left(^{*}\right)$ per determinare tutti gli spazii a tre dimensioni con un gruppo continuo di movimenti non illuminano abbastanza sul problema generale: uno dei risultati del presente lavoro è appunto quello di dare un metodo generale per risolvere con sole quadrature il problema, e che, applicato al caso particolare di spazii a tre dimensioni, permetterebbe di trovare rapidamente $i$ risultati del prof. Bianchi. Ma nè il metodo generale, nè il metodo del prof. Bianchi generalizzato possono poi condurre senza una interminabile serie di calcoli all'effettiva determinazione di tali spazii quando il numero delle loro dimensioni è maggiore di tre: la Memoria seguente svolgerà per il caso di quattro dimensioni un metodo assai più rapido, e comodo.

Lo studio infine dei sottogruppi finiti discontinui di movimenti ammessi da tali spazii condurrà, fra l'altro, a notevoli rappresentazioni degli spazii dei tipi (VIII) e (IX) del prof. Bianchr sulla sfera e sulla pseudosfera.

(*) Memorie della Società Italiana delle Scienze, 1897: Sugli spazii a tre dimensioni, che ammettono, ecc. Questa Memoria sarà in seguito indicata con (A). Avverto pure che la classica Theorie der Transformationsgruppen del LIE-ENGeL sarà indicata in seguito soltanto col nome di Lif. 
Un altro risultato del presente lavoro che spero possa presentare qualche interesse, è di dare le condizioni necessarie e sufficienti affinchè un gruppo si possa considerare come gruppo di movimenti: condizioni, da cui si può trarre qualche proprietà degna di nota per taluni di tali gruppi.

Mi sia permesso infine di ringraziare qui vivamente l'illustre prof. LevrCivita, che acconsenti a leggere questo lavoro, prima che fosse pubblicato.

$\S 1$. Noi vogliamo trovare anzitutto dei teoremi generali relativi alla ricerca degli spazii che ammettono un gruppo continuo di movimenti: principio della nostra ricerca sarà di distinguere il problema in due parti: la prima relativa alla ricerca dei gruppi, che si possono considerare come gruppi di movimenti di uno spazio, la seconda relativa alla determinazione di uno spazio, di cui sia noto il gruppo corrispondente.

Il teorema fondamentale della prima parte delle nostre ricerche è il seguente :

Se un gruppo $G_{m}$ trasforma in sè uno spazio $S_{n}$, e se le varietì minime invarianti sono delle $V_{n-k}$, ognuna di queste $V_{n-k}$ è pure trasformata in sè da un gruppo proprio ad $m$ parametri, $e$ il gruppo $G_{m}$ si pù̀, con un opportuno cangiamento di variabili, ridurre a un gruppo transitivo su $n-k$ variabili $\left({ }^{*}\right)$.

Per dimostrare il nostro teorema, prendiamo una varietà $V_{n-1}^{(1)}$ composta di $V_{n-k}$ invarianti, tale cioè che per ogni punto di $V_{n-1}^{(1}$ passi almeno una $V_{n-k}$ invariante contenuta nella $V_{n-1}^{(1)}$ e scegliamo come coordinata $(n-k+1)^{\text {esima }} \mathrm{di}$ un punto $A$ di $S_{n}$ la distanza geodetica $y_{n-k+1}$ dal punto stesso alla $V_{n-1}^{(1)}$ in discorso. Le $y_{n-k+1}=$ cost. sono delle varietà $V_{n-1}$ geodeticamente parallele alla $V_{n-1}^{(1)}$ e quindi sono come la $V_{n-1}^{(1)}$ invarianti, ossia composte di varieta minime invarianti. Immaginiamo ora nella $V_{n-1}^{(1)}$ scelta una $V_{n-4}^{(1)}$ composta pure di $V_{n-k}$ invarianti e consideriamo la varietà $V_{n-1}^{(2)}$ generata dalle geodetiche dello spazio ambiente normali a $V_{n-1}^{(1)}$ nei punti di $V_{n-2}^{(1)}$. Le trasformazioni di $G_{m}$ mutando la $V_{n-1}^{(1)}$ e la $V_{n-2}^{(1)}$ in sè stesse, e le geodetiche normali a $V_{n-1}^{(1)}$ pure

(*) Come seppi, dopo che questo lavoro era gia terminato, in una Memoria, che io non conoscevo, del Bisconcins, inserita nel Nuovo Cimento (Aprile 1901): Su una classifcasione dei problemi dinamici, questo teorema era gia stato enunciato: peró la dimostrazione del BIsconcini in realtà vorrebbe invece provare che «ogni gruppo continuo con $p$ trasformazioni infinitesime indipendenti si può ridurre a un gruppo su $p$ variabili » ed è perciò completamente sbagliata (cfr. LIE; vol. I, § 109), perchè questa proposizione non $\dot{e}$ vera in generale. Le mie consilerazioni rendono rigorosi gli altri bei risultati del $\mathrm{BI}$ SCONCINI, 
in geodetiche normali a $V_{n-1}^{(1)}$, è ben certo che la $V_{n-1}^{(2)}$ è una varietà invariante, ossia che una $V_{n-k}$ invariante che passa per un sno punto giace tutta in essa. La $V_{n-1}^{()}$dovrà quindi tagliare ogni varietà $y_{n-k+1}=$ cost. pure in varietà invarianti. Posto questo, osserviamo che per determinare la posizione di un punto di $S_{n}$, basterà conoscerne la proiezione geodetica $A^{(1)}$ su $V_{n-1}^{(1)}$ e la coordinata $y_{n-k+1}$.

E noi siamo così ricondotti per determinare un sistema di coordinate in $S_{n}$, a cercare un sistema di coordinate per la $V_{n-1}^{(1)}$, la quale varietà è, come lo spazio ambiente, trasformata in sè dal gruppo $G_{m}$. E noi perciò opereremo sulla $V_{n-1}^{(1)}$, come abbiamo operato in $S_{n}$. Noi determineremo cioè la posizione di un punto $A^{(1)}$ di $V_{n-1}^{(1)}$ nel seguente modo: Prenderemo una varietà $V_{n-2}^{(1)}$, tutta contenuta in $V_{n-1}^{(1)}$ e composta di varietà minime invarianti e individueremo un punto $A^{(1)}$ di $V_{n-1}^{(1)}$ dando la sua distanza geodetica $y_{n-k+2}$ dalla $V_{n-2}^{(1)}$ (cioè la distanza misurata sulla geodetica di $V_{n-1}^{(1)}$ tirata dal punto in discorso normalmente alla $V_{n-2}^{(1)}$ e la posizione della sua proiezione $A^{(2)}$ sulla $V_{n-2}^{(1)}$ stessa. E come coordinata $(n-k+2)^{\text {esima }}$ di un punto qualunque $A$ di $S_{n}$ prenderemo appunto la $y_{n-k+2}$ testè definita, relativa alla sua proiezione $A^{(1)}$ sulla $V_{n-1}^{(1)}$. Ora, per un ragionamento già usato, è ben chiaro che le $y_{n-k+2}=$ cost. sono nella $V_{n-1}^{(1)}$ varietà invarianti, perchè geodeticamente parallele alla $V_{n-2}^{(1)}$ entro $V_{n-1}^{(1)}$; e per quanto abbiamo notato saranno pure invarianti quelle varietà di $S_{n}$ generate dalle geodetiche di $S_{n}$ normali a $V_{n-1}^{(1)}$ nei punti di una di queste varietà $y_{n-k+2}=$ cost., ossia anche in tutto lo spazio ambiente $S_{n}$ le $y_{n-k+2}=$ cost. sono varietà (a $n-1$ dimensioni) invarianti.

Ci siamo dunque ridotti a determinare la posizione di un punto $A^{(2)}$ di $V_{n-2}^{(i)}$. E anche qui, procedendo con lo stesso metodo, prenderemo una $V_{n \rightarrow 3}^{(1)}$, contenuta nella $V_{n-2}^{(1)}$, e invariante; e definiremo la posizione di un punto $A^{(2)}$ di $V_{n-2}^{(1)}$ dandone la distanza geodetica $y_{n-k+3}$ (cioè misurata lungo una geodetica di $V_{n-2}^{(1)}$ normale alla $V_{n-3}^{(1)}$ ) dalla $V_{n-3}^{(1)}$ stessa e la posizione della sua proiezione $A^{(3)}$ sulla $V_{n-3}^{(1)}$. E definiremo poi come coordinata $(n-k+3)^{\text {esima }}$ di un punto qualunque $A$ di $S_{n}$ la $y_{n-k+3}$, testè definita, relativa alla proiezione $A^{(2)}$ su $V_{n-2}^{(1)}$ della proiezione $A^{(1)}$ del punto $A$ sulla $V_{n-1}^{(1)}$. Per una osservazione già usata, le $y_{n-k+3}=$ cost. (che nella $V_{n-2}^{(1)}$ sono varietà invarianti, perchè geodeticamente parallele alla $V_{n-3}^{(1)}$ ) sono varietà pure invarianti in $V_{n-1}^{(1)}$ e quindi anche nello spazio ambiente $S_{n}$.

Così si prosegue fino ad arrivare a una $V_{n-l+1}^{(1)}$ invariante, in cui la posizione di un suo punto qualunque $A^{(k-1)}$ si definisce dandone la distanza 
geodetica $y_{n}$ da una $V_{n-k}^{(1)}$ invariante della $V_{n-k+1}^{(1)}$ e la posizione della sua proiezione $A^{(k)}$ sulla $V_{n-k}^{(1)}$ stessa. E si definisce come coordinata $n^{e s i m a}$ di un punto $A$ di $S_{n}$ la $y_{n}$, testè definita, relativa a quel punto $A^{\left(k_{-1}\right)}$ di $V_{n-k+1}^{(1)}$, a cui si perviene mediante le successive proiezioni considerate. Nella $V_{n-k}^{(1)}$ si assume poi un sistema qualsiasi di coordinate $y_{1}, y_{2}, \ldots y_{n-k}$; e per le prime $n-k$ coordinate di un punto $A$ di $S_{n}$ si scelgono appunto le $y_{1} \ldots y_{n-k}$ relative al corrispondente punto $A^{(k)}$ di $V_{n-k}^{(1)}$ a cui si arriva mediante successive proiezioni.

Le osservazioni da noi fatte ci dicono senz'altro che le

$$
y_{n-k+i}=\text { cost. } \quad(i=1,2, \ldots, k)
$$

sono in $S_{n}$ varietà invarianti, e che quindi le

$$
y_{n-k+1}=\text { cost. }, \ldots, y_{n}=\text { cost. }
$$

sono precisamente le varietà minime invarianti.

Quale aspetto assume ora il nostro gruppo con questo sistema di varia* bili? Intanto, siccome le $y_{n-k+i}=$ cost. sono, come si disse, varietà invarianti è certo che il nostro gruppo non trasforma le $y_{n-k+1}, y_{n-k+2}, \ldots y_{n}$ ossia che se le trasformazioni di $G_{m}$ sono date dalle:

$$
y_{t}^{\prime}=f_{t}\left(y_{1} \ldots y_{n-k} y_{n-k+1} \ldots y_{n}\right) \quad(t=1,2, \ldots, n)\left(^{*}\right)
$$

(dove le $f_{t}$ conterranno $m$ parametri arbitrarij, sarà certamente:

$$
f_{n-k+i}=y_{n-k+i} \quad(i=1,2, \ldots k) .
$$

Ma noi diciamo di più che le $f_{1} \ldots f_{n-k}$ non contengono $y_{n-k+1} \ldots y_{n}$ e dipendono cioè soltanto (oltre che dai parametri del gruppo) dalle variabili $y_{1} \ldots y_{n-k}$.

In altre parole: noi dimostreremo che le prime $" n-k$ " coordinate $y_{1}^{\prime} \ldots y_{n-k}^{\prime}$ di quel punto $A_{0}$, dove un punto $A$ di coordinate $y_{1} \ldots y_{n-k}$ $y_{n-k+1} \ldots y_{n}$ ̀̀ condotto da una trasformazione $\Gamma$ qualsiasi di $G_{m}$ non dipendono dai valori di $y_{n-k+1} \ldots y_{n}$, ossia che $\Gamma$ conduce il punto che ha per prime coordinate proprio $y_{1} \ldots y_{n-k}$, e che ha tutte le altre coordinate uguali a zero, nel punto, le cui prime coordinate sono $y_{1}^{\prime} \ldots y_{n-k}^{\prime}$ e di cui tutte le altre coordinate sono nulle.

$\mathrm{O}$ in altre parole io dimostrerò che se $A^{(1)}$ è la proiezione di un punto $A$ di $S_{n}$ sulla $V_{n-1}^{(1)}, A^{(2)}$ la proiezione di $A^{(1)}$ sulla $V_{n-2}^{(1)}, \ldots, A^{(k)}$ quella di

(*) Dove indichiamo con $y^{\prime}{ }_{t}$ i valori trasformati delle $y_{t}$. 
$A^{(k-1)}$ sulla $V_{n-k}^{(1)}$, e se una trasformazione $\Gamma$ di $G_{m}$ conduce i punti $A$ ed $A^{(k)}$ rispettivamente in $B$ e in $B^{\prime}$, il punto $B^{\prime}$ coincide con quel punto $B^{(k)}$ di $V_{n-k}^{(1)}$ che si dedurrebbe da $B$ con le accennate successive proiezioni. $\mathrm{E}$ infatti, essendo $V_{n-1}^{(1)}$ invariante, la geodetica per $A$ normale a $V_{n-1}^{(1)}$ sarà portata nella geodetica per $B$ normale a $V_{n-1}^{(1)}$ e quindi anche $A^{(1)}$ sarà portato nella proiezione $B^{(1)}$ di $B$ su $V_{n-1}^{(t)}$; così $A^{(2)}$ sarà portato nella proiezione $B^{(2)}$ di $B^{(1)}$ su $V_{n-2}^{(1)}$ ecc.; e così continuando arriveremo alla dimostrazione dell'asserto, cioè che $\Gamma$ conduce $A^{(k)}$ in $B^{(k)}$ e che quindi i punti $B^{\prime}, B^{(k)}$ coincidono.

$\S 2$. Posto questo, noi ora procederemo a dare alcune proprietà di un gruppo, che sono condizioni necessarie e sufficienti, affinchè esso si possa considerare come un gruppo di movimenti. E comincieremo anzitutto dalla considerazione dei gruppi a trasformazioni infinitesime linearmente indipendentj; e rammenteremo che il Prof. Bianchi dimostrò (e più sotto io ne darò una dimostrazione intuitiva, che ne spiega l'intima ragione di essere) che ogni gruppo $G_{n}$ transitivo su $n$ variabili è ammesso da qualche spazio a $n$ dimensioni; noi dimostreremo anzi che ciò è vero per qualsiasi gruppo a $n$ variabili con trasformazioni infinitesime linearmente indipendenti. Noi per ora, a proposito di questi gruppi, non possiamo che dare il teorema seguente: Condizione necessaria e sufficiente affinchè un gruppo $G_{m}$ a trasformazioni infinitesime linearmente indipendenti su $n+m$ variabili si possa considerare come gruppo di movimenti di uno spazio ad $n+m$ dimensioni è che il gruppo sia simile a un gruppo semplicemente transitivo su m lettere; ciò che si può anche esprimere dicendo che $G_{m}$ 'deve essere un sottogruppo di un gruppo semplicemente transitivo con $n+m$ lettere ed $n+m$ parametri $(*)$.

La condizione è evidentemente necessaria; infatti il gruppo $G_{m}$ ha come varietà minime invarianti delle $V_{m}$ : e, se $S_{n+m}$ è uno spazio che ammette $G_{m}$, si vede tosto prendendo in esso quel sistema di coordinate, che fu definito al paragrafo precedente che il gruppo $G_{m}$ sarà trasformato in un gruppo simile che opera transitivamente su' $m$ lettere. Che questa condizione sia anche sufficiente, si dimostra facilmente così: Se $G_{m}$ è simile a un gruppo che opera transitivamente su $m$ variabili $y_{1} y_{2} \ldots y_{m}$, si introducano come coordinate appunto le $y_{1} y_{2} \ldots y_{m}$ insieme ad altre $n$ funzioni qualsiasi delle coordinate iniziali $y_{m+1} \ldots y_{m+n}$ tali che $y_{1} \ldots y_{m} y_{m+1} \ldots y_{m+n}$ siano indipendenti tra

(*) Ricordiamo peró esplicitamente (a scanso di ogni equivoco) che, come faremo vedere più tardi, queste condizioni sono sempre soddisfatte da un tale gruppo. 
loro. Le trasformazioni infinitesime di $G_{m}$ insieme alle trasformazioni $\frac{\partial}{\partial y_{n+1}}, \ldots, \frac{\partial}{\partial y_{m+n}}$ genereranno un gruppo $\Gamma_{m+n}$, semplicemente transitivo, e di cui $G_{m}$ è un sottogruppo. Per il citato teorema del prof. Bianchi $\Gamma_{m+n}$ è ammesso da qualche $S_{m+n}$, che ammetterà perciò anche $G_{m}$.

Nella seconda parte di questa dimostrazione abbiamo visto incidentalmente che se un $G_{m}$ è simile a un gruppo transitivo su $m$ lettere, esso ̀̀ anche sottogruppo di un $\Gamma_{m+n}$ transitivo in $S_{m+n}$. Viceversa se $G_{m}$ è sottogruppo di un $\Gamma_{m+n}$ transitivo in $S_{m+n}$, esso sarà certo un gruppo di movimento di ciascuno degli spazii, che ammettono $\Gamma_{m+n}$ e quindi, per quanto abbiamo dimostrato, sarà simile a un gruppo semplicemente transitivo su $m$ lettere.

Noi più tardi dimostreremo che ogni gruppo a trasformazioni infinitesime linearmente indipendenti può essere considerato come gruppo di movimenti : il presente teorema ci darà perciò alcune interessanti propriełà generali di gruppi siffatti; ciò che permette di ngevolarne di molto la ricerca.

$\S$ 3. Abbiamo così studiato i gruppi a trasformazioni infinitesime linearmente indipendenti, che possono essere considerati (tutti) come gruppi di movimenti e abbiamo visto che in fin dei conti essi si riducono tutti ai gruppi transitivi, in cui il numero dei parametri eguaglia quello delle variabili. Ora ci proporremo la questione di riconoscere quando un gruppo a trasformazioni infinitesime linearmente dipendenti può essere considerato come gruppo di movimenti. Noi ora daremo una prima risoluzione di questo problema, avvertendo però che noi vi ritorneremo più oltre, con altri metodi, e ottenendo una risoluzione più elegante ed intuitiva.

$\Lambda$ nzitutto osserveremo che per il teorema fondamentale del $\S 1$, se il nostro gruppo è un gruppo $G_{n+m}$ a $" n+m$ " parametri con sole $n$ trasformazioni infinitesime linearmente indipendenti, esso (avendo delle $V_{n}$ per varietà minime invarianti) sarà certo simile a un gruppo transitivo su $n$ variabili, e si potrà considerare come gruppo di movimenti di una varietà ad $n$ dimensioni. Noi supporremo senz'altro che il gruppo sia già stato ridotto sotto questa forma, che diremo forma normale del gruppo, con un opportuno cangiamento di variabili. Siano dunque

$$
X_{t}=\sum_{r=1}^{n} \xi_{r}^{(t)}\left(x_{1} \ldots x_{n}\right) \frac{\partial}{\partial x_{r}} \quad(t=1,2, \ldots n+m)
$$

le trasformazioni infinitesime del nostro gruppo $G_{n+m}$ e sia

$$
d s^{2}=\sum_{i, k}^{n} a_{i k} d x_{i} d x_{k}
$$


l'elemento lineare di uno spazio $S_{n}$ che ammetta $G_{n+m}$. Le formule di KiLLive danno che

$$
\varliminf_{r}^{\mathbf{\Sigma}} \xi_{r}^{(l)} \frac{\partial a_{i k}}{\partial x_{r}}+\underset{r}{\mathbf{\Sigma}}\left(a_{i r} \frac{\partial \xi_{r}^{(l)}}{\partial x_{k}}+a_{k r} \frac{\partial \xi_{r}^{(l)}}{\partial x_{i}}\right)=0 \quad(l, r=1,2, \ldots, n) .
$$

E noi le scriveremo, ponendo

sotto la forma

$$
a_{i k}^{(l)}=\sum_{r}\left(a_{i r} \frac{\partial \xi_{r}^{(l)}}{\partial x_{k}}+a_{k r} \frac{\partial \xi_{r}^{(l)}}{\partial x_{i}}\right)
$$

$$
\sum_{r} \xi_{r}^{(l)} \frac{\partial a_{i k}}{\partial x_{r}}+a_{i k}^{(i)}=0, \quad \text { ossia } \quad X_{l}\left(a_{i k}\right)+a_{i k}^{(i)}=0 .
$$

Il prof. Branchi osservò che in virtù delle (1)' si ha che

$$
X_{l}\left[X_{t}\left(a_{i k}\right)+a_{i k}^{(t)}\right]-X_{t}\left[X_{l}\left(a_{i k}\right)+a_{i k}^{i l}\right] \quad(l, t=1,2, \ldots, n+m)
$$

è identicamente nullo: ciò che del resto è intuitivo perchè se un $S_{n}$ ammette due trasformazioni infinitesime $X_{l}, X_{t}$ esso deve ammettere anche la $\left(X_{l}, X_{t}\right)$. Per ipotesi $m$ delle trasformazioni infinitesime del gruppo sono combinazione lineare delle altre $n$, le quali sono poi linearmente indipendenti. Noi ammetteremo che queste ultime sieno le $X_{1}, X_{2}, \ldots, X_{n}$ e porremo, indicando con $\varphi_{i}^{(i)}$ delle funzioni di $x_{1}, x_{2}, \ldots x_{n}$

$$
X_{n+i}=\sum_{l=1}^{n} c_{i l}^{(i)} X_{l} \quad(i=1,2, \ldots m) .
$$

E il sistema delle equazioni di KiLurna sarà da noi scritto nella forma:

$$
\begin{gathered}
X_{l}\left(a_{i k}\right)+a_{i k}^{(i)}=0 \quad(l=1,2, \ldots n) \\
X_{n+t}\left(a_{i k}\right)-\sum_{l=1}^{n} \varphi_{l}^{(t)} X_{l}\left(a_{i k}\right)+a_{i k}^{(i+t)}-\sum_{i=1}^{n} \varphi_{l}^{(t)} a_{i k}^{(i)}=0 \quad(t=1,2, \ldots m) .
\end{gathered}
$$

Ciò è chiaramente lecito perchè se alla (4) aggiungiamo

$$
\sum_{l=1}^{n} \varphi_{l}^{(i)}\left[X_{l}\left(a_{i k}\right)+a_{i k}^{(l)}\right]
$$

somma evidentemente nulla in causa delle (3), otteniamo appunto l'equazione di Kiluina relativa a $X_{n+t}$ ed a $a_{i \hbar}$, come è scritta nella (1)', E ora poichè è identicamente

$$
X_{n+t}\left(a_{i k}\right)-\sum_{l=1}^{n} \varphi_{l}^{(i)} X_{l}\left(a_{i k}\right)=0
$$


le (4) diventano

ossia

$$
a_{i k}^{(n+t)}-\sum_{i=1}^{n} \varphi_{l}^{(t)} a_{i k}^{(l)}=0
$$

$$
\left.\begin{array}{c}
\sum_{r=1}^{n} a_{i r}\left[\mathbf{\sum} \frac{\partial\left(\varphi_{l}^{(t)} \xi_{r}^{(i)}\right)}{\partial x_{k}}-\sum_{\tau} \varphi_{l}^{(t)} \frac{\partial \xi_{r}^{(i)}}{\partial x_{k}}\right]+ \\
+\sum_{r=1}^{n} a_{k r}\left[\mathbf{\Sigma} \frac{\partial\left(\psi_{l}^{(t)} \xi_{r}^{(i)}\right)}{\partial x_{i}}-\sum_{l} \varphi_{l}^{(t)} \frac{\partial \xi_{r}^{(i)}}{\partial x_{i}}\right]=0
\end{array}\right\}
$$

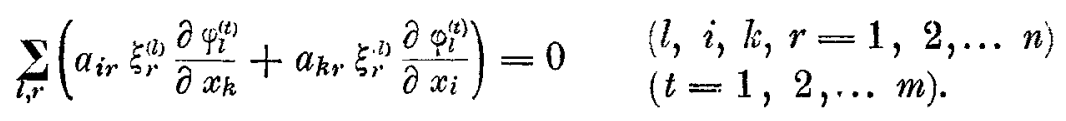

Il nostro sistema di equazioni resta cos̀̀ mutato nel sistema di equazioni formato dalle (3) e dalle $(4)^{\prime \prime \prime}$. Ora dalle (3), essendo il determinante delle $\xi_{r}^{(t)}(r, l=1,2, \ldots n)$ differente da zero si possono trarre le derivate delle $a_{i k}$ in funzione delle $a_{i k}$ stesse; e, per l'osservazione testè citata del professore Bianch si vede che affinchè il sistema delle $(3),(4)^{\prime \prime \prime}$ sia integrabile basta che

1. ${ }^{\circ}$ Le $(4)^{\prime \prime \prime}$ considerate come equazioni lineari algebriche per le $a_{i k}$ ammettono in un punto almeno una soluzione (che ci darà $i$ valori iniziali delle $a_{i k}$ ). Si dovrà poi solo esaminare (volendoci restringere a spazii reali) se le solite disuguaglianze sono soddisfatte.

2. ${ }^{\circ}$ I primi membri delle $(4)^{\prime \prime \prime}$ derivate rispetto a $x_{1}, x_{2}, \ldots x_{n}$ successivamente dieno per le (3) e le (4)" stesse risultati identicamente nulli. Questa seconda condizione equivale anche (essendo il determinante delle $\xi_{\text {, }}^{\text {, }}$ diverso da zero) all'altra condizione che applicando ai primi membri delle $(4)^{\prime \prime \prime}$ le $X_{1} \ldots X_{n}$ si ottenga in virtù delle (3) e delle $(4)^{\prime \prime \prime}$ stesse un risultato identicamente nullo.

Noi ora vogliamo far vedere che questa seconda condizione è sempre soddisfatta.

Infatti per l'osservazione del prof. Braschi è sempre per le (3) e le (4)"' identicamente :

$$
\begin{aligned}
X_{l}\left[X_{n+t}\left(a_{i k}\right)+a_{i k}^{(n+t)}\right]-X_{n+t}\left[X_{l}\left(a_{i k}\right)+a_{i k}^{(i)}\right]=0 \\
{[t=1,2, \ldots m][i, k, l=1,2, \ldots n] }
\end{aligned}
$$

ossia

$$
X_{l}\left[X_{n+t}\left(a_{i k}\right)\right]-X_{n+t}\left[X_{l}\left(a_{i k}\right)\right]+X_{i}\left(a_{i k}^{(n+t)}\right)-X_{n+t}\left(a_{i k}^{(i)}\right)=0 .
$$


Si ha pure identicamente:

$$
\begin{array}{r}
X_{l}\left[X_{r}\left(a_{i k}\right)\right]-X_{r}\left[X_{l}\left(u_{i k}\right)\right]+X_{l}\left[a_{i k}^{(i k}\right]-X_{r}\left[a_{i k}^{(i)}\right]=0 \\
(l, r, i, k=1,2, \ldots, n)
\end{array}
$$

ossia

$$
\varphi_{i r}^{(t)} X_{l}\left[X_{r}\left(a_{i k}\right)\right]-\varphi_{r}^{(t)} X_{r}\left[X_{l}\left(a_{i k}\right)\right]+\varphi_{r}^{(t)} X_{l}\left(a_{i k}^{(p)}\right)-\varphi_{r}^{(t)} X_{r}\left(a_{i k}^{(i)}\right)=0
$$

ossia :

$$
\begin{aligned}
& X_{l}\left[\varphi_{r}^{(t)} X_{r}\left(a_{i k}\right)\right]-X_{l}\left(\varphi_{r}^{(t)}\right) X_{r}\left(a_{i k}\right)-\varphi_{r}^{(t)} X_{r}\left[X_{l}\left(a_{i k}\right)\right]+ \\
& +X_{l}\left[\varphi_{r}^{(t)} a_{i k}^{(i)}\right]-X_{l}\left[\varphi_{r}^{(t)}\right] a_{i k}^{(r)}-\varphi_{r}^{(t)} X_{r}\left(a_{i k}^{(l)}\right)=0 .
\end{aligned}
$$

E, poichè per le (3)

$$
X_{r}\left(a_{i k}\right)+a_{i k}^{(i)}=0
$$

sarà pure identicamente (in virtù delle $(3)$ e delle $(4)^{\prime \prime \prime}$ )

$$
X_{l}\left[y_{r}^{(t)} X_{r}\left(a_{i k}\right)\right]-\varphi_{r}^{(t)} X_{r}\left[X_{l}\left(a_{i k}\right)\right]+X_{l}\left[\varphi_{r}^{(t)} a_{i k}^{(r)}\right]-\varphi_{r}^{(t)} X_{r}\left(a_{i k}^{(l)}\right)=0
$$

e quindi anche identicamente:

$$
\sum_{r=1}^{n}\left\{X_{l}\left[\varphi_{r}^{(t)} X_{r}\left(a_{i k}\right)\right]-\varphi_{r}^{(i)} X_{r}\left[X_{l}\left(a_{i k}\right)\right]+X_{l}\left[\psi_{r}^{(i)} a_{i k}^{(r)}\right]-\varphi_{r}^{(t)} X_{r}\left(a_{i k}^{(l)}\right)\right\}=0 \ldots
$$

Sottraendo dalla (5) la (6) troviamo l'identità :

$$
\begin{aligned}
X_{l}\left[X_{n+t}\left(a_{i k}\right)\right. & \left.-\sum_{r=1}^{n} \varphi_{r}^{(t)} X_{r}\left(a_{i k}\right)\right]-\left[X_{n+t}-\sum_{r=1}^{r} \dot{\bar{c}}_{r}^{n} X_{r}^{(t)}\right]\left(X_{l}\left(a_{i k}\right)\right)+ \\
& +X_{l}\left[a_{i k}^{(n+t)}-\sum_{r=1}^{n} \psi_{r}^{(t)} a_{i k}^{(t)}\right]-\left[X_{n+t}-\sum_{r=1}^{r} \varphi_{r}^{(t)} X_{r}\right]\left(a_{i k}^{(i)}\right)=0 .
\end{aligned}
$$

E poichè è identicamente:

$$
X_{n+t}-\sum_{r=1}^{n} \varphi_{r}^{(t)} X_{r}=0
$$

troveremo senz'altro identicamente :

$$
X_{l}\left(a_{i l}^{(n+t)}-\sum_{,=1}^{n} \varphi_{r}^{(t)} a_{i n}^{(n)}\right)=0 \quad(l=1,2, \ldots n)
$$

che è appunto quarto si voleva dimostrare. (Si ricordi che $\mathrm{i}$ primi membri delle $(4)^{\prime}$ e delle $(4)^{\prime \prime \prime}$ sono identici.)

Prima ora di venire a parlare della $1 .^{a}$ condizione vogliamo fare un'osservazione generale. 
Se un gruppo $\Gamma$ qualsiasi si può considerare come gruppo di movimenti di uno spazio a $n$ dimensioni, esso può essere anche considerato come gruppo di movimenti di uno qualche spazio a un numero $n+t$ di dimensioni $(t$ intero qualsiasi).

Supposto infatti il gruppo ridotto a forma normale in $n$ o meno variabili, e se

$$
d s^{2}=\sum_{i, k} a_{i k} d x_{i} d x_{k} \quad(i, k=1,2, \ldots n)
$$

è l'elemento di uno spazio a $n$ dimensioni che ammette il nostro gruppo, allora, come tosto si verifica

$$
\begin{aligned}
& d s^{2}=\sum_{i, k} a_{i k} d x_{i} d x_{k}+\sum_{l m} a_{l m} d x_{l} d x_{m} \\
& (i, k=1,2, \ldots n)(l, m=n+1, \ldots n+t)
\end{aligned}
$$

dove le $a_{l m}$ non dipendono dalle $x_{i}, x_{2}, \ldots x_{n}$ è l'elemento lineare di uno spazio a $n+t$ dimensioni che ammette il nostro gruppo.

Dungue :

Affinchè un dato gruppo, gì̀ ridotto a forma normale in $n$ variabili, possa essere ammesso come gruppo di movimenti da uno spazio (che naturalmente non potrà avere meno di $n$ dimensioni) $\grave{e}$ condizione necessaria $e$ sufficiente che le $(4)^{\prime \prime \prime}$ considerate come equazioni lineari algebriche nelle $a_{i k}$ sieno in un punto risolubili rispetto alle $a_{i k}$. Dunque questa condizione si esprime semplicemente con l'annullarsi di una matrice.

Ora osserviamo il primo membro delle (4)"' e vediamo tosto che il termine $\sum_{l, r} a_{i r} \xi_{r}^{(!)} \frac{\partial \varphi_{l}^{(t)}}{\partial x_{k}}$ è un termine del determinante che si ottiene moltiplicando con le note regole, in modo opportuno il determinante delle $a_{i k}$ per il determinante delle $\xi_{r}^{(l)}$ e per l'Iacobiano delle $\xi^{(l)}$. Quindi abbiamo:

Condizione necessaria e sufficiente affinchè il nostro gruppo già ridotto a forma normale possa essere considerato come gruppo di movimenti è che si possa trovare un determinante non nullo simmetrico $\left|a_{i k}\right|$, il prodotto del quale per il determinante $\left|\xi_{r}^{(l)}\right|(r, l=1,2, \ldots n)$ e per l'Iacobiano delle ${ }_{i}^{(l)}(r=1,2, \ldots n)$ ottenuto in modo opportuno con le note regole sia, almeno in un punto, semisimmetrico. $E$ ciò per ciascun valore particolare di $t$.

Se ora $n$ è dispari, il determinante semisimmetrico così ottenuto è nullo; e poichè $\left|a_{i k}\right|==_{1}^{\prime}=0\left|\xi_{r}^{\prime \prime}\right|==0$, sarà $\frac{\partial\left(\varphi_{1}^{\prime t} \varphi_{2}^{(t)} \ldots \varphi_{n}^{(t)}\right)}{\partial\left(x_{1} x_{2} \ldots x_{n}\right)}=0$ ossia le $\varphi_{1}^{(t)} \ldots \varphi_{n}^{(t)}$ non sono funzioni indipendenti. 
Quindi in particolare:

Se un $S_{n}$ ammette un $G_{n+1}$ transitivo, esiste certamente nel caso che $n$ sia dispari, qualche trasformazione infinitesima permutabile con $G_{n+1}$.

$\S 4$. Sia ora dato un gruppo, già ridotto sotto forma normale, cioè con tante trasformazioni infinitesime linearmente indipendenti quante sono le variabili su cui opera il gruppo. Noi vogliamo esaminare come si semplificano in questo caso le equazioni di KiLLina. Siano

$$
X_{l}=\sum_{r=1}^{n} \xi_{r}^{(l)}\left(x_{1} x_{2} \ldots x_{n}\right) \frac{\partial}{\partial x_{r}} \quad(l=1,2, \ldots, n+m)
$$

le trasformazioni infinitesime del gruppo e sia

$$
d s^{2}=\sum_{i, k} a_{i k} d x_{i} d x_{k} \quad(i, k=1,2, \ldots, n+t)
$$

l'elemento lineare di uno spazio a $" n+t$ " dimensioni che ammetta il nostro gruppo.

Se noi scriviamo le equazioni di Killiva noi riconosciamo facilmente:

1. Per le $a_{i k}(i, k=1,2, \ldots n)$ queste equazioni assumono proprio la stessa forma, come se noi volessimo cercare gli spazii ad $n$ dimensioni ammessi dal gruppo.

2. ${ }^{\circ}$ Per le $a_{i k}(i, k=n+1, n+2, \ldots, n+t)$ le equazioni di KILLING diventano semplicemente:

$$
\sum_{i=1}^{n} \xi_{r}^{l_{l}} \frac{\partial a_{i k}}{\partial x_{r}}=0
$$

E poichè delle $X_{l}$ proprio $n$ sono linearmente indipendenti, si ha semplicemente:

$$
\frac{\partial a_{i k}}{\partial x_{r}}=0 \quad(r=1,2, \ldots, n)
$$

e le equazioni di KiLLING dicono soltanto che le $\alpha_{i k}$ in discorso sono funzioni soltanto di $x_{n+1}, \ldots, x_{n+t}$.

3. ${ }^{\circ}$ Per le $a_{i k}(i \leq n, k>n)$ le equazioni di KuLLiva diventano:

$$
\sum_{r} \xi_{r}{ }^{l} \frac{\partial a_{i k}}{\partial x_{r}}+\sum_{r} a_{k r} \frac{\partial \xi_{r}^{i}}{\partial x_{i}}=0
$$

che è un sistema di equazioni che non contiene nessuna $a_{i k}$ del primo o del secondo tipo. Anzi questo sistema di equazioni si scinde per ciascun valore di $k$ in $t$ sistemi di equazioni distinti, l'uno relativo alle $a_{i, n+1}$, il secondo alle $a_{i, n+2}$, ecc. $(i=1,2, \ldots, n)$. 
Le equazioni relative alle $a_{i k}(i, k \leq n)$ si semplificano, ossia si possono ridurre a un numero minore di incognite mediante le equazioni lineari $(4)^{\prime \prime \prime}$, mentre nello stesso tempo per ciascuna delle $a_{i k}$ da determinarsi si ottengono soltanto $n$ equazioni. Queste osservazioni riescono utili specialmente per il calcolo effettivo degli elementi lineari da determinarsi.

$\S 5$. Ora noi vogliamo dimostrare, servendoci dei risultati finora ottenuti, il seguente teorema fondamentale:

Con la sola risoluzione di equazioni algebriche si possono determinare tutti $i$ gruppi, che si possono considerare come gruppi di movimenti, trovando le loro trasformazioni infinitesime; con sole quadruture si possono determinare le trasformazioni finite di tali gruppi, e gli elementi lineari degli spazii loro corrispondenti.

La dimostrazione di questo teorema è semplicissima: basta a tale scopo la semplice osservazione che ogni gruppo continuo, che si possa considerare come gruppo di movimenti, si può, con un cangiamento di variabili ridurre sotto forma normale, cioè si può far sì che possegga tante trasformazioni infinitesime linearmente indipendenti quante sono le variabili su cui opera effettivamente: in altre parole si può ridurre a essere transitivo sulle variabili che esso effettivamente trasforma. Ora noi sappiamo che con la risoluzione di sole equazioni algebriche si possono trovare tutte le possibili composizioni realmente distinte dei gruppi a un numero qualsiasi di parametri. Di più (Lie, Kap. 27, 29) con la sola risoluzione di equazioni algebriche si possono trovare tutti i gruppi continui transitivi, appena se ne conosca la composizione, e non si considerino distinti due gruppi simili. È così dimostrata senz'altro la prima parte del nostro teorema. Esaminando la forma delle trasformazioni infinitesime dei nostri gruppi si potrebbe dimostrare anche la terza parte del nostro teorema; ma il procedimento più breve a tale scopo è il seguente: Si ricordi anzitutto che con sole quadrature si possono trovare le equazioni finite del nostro gruppo, perchè il gruppo è transitivo (Lie, l. c.), ciò che intanto dimostra la seconda parte del nostro teorema. Allora distinguiamo due casi : secondoche le dimensioni dello spazio da determinarsi sono in numero uguale o maggiore del numero delle variabili, su cui il nostro gruppo, che col procedimento precedente è stato evidentemente ottenuto sotto forma normale, opera effettivamente. Cominciamo a trattare il primo caso: sia cioè uguale il numero $n$ delle dimensioni dello spazio da determinarsi e delle variabili su cui opera (transitivamente) il gruppo. Sia $A$ un punto generico dello spazio e siano $a_{i k}^{\text {in }}$ valori qualsiasi che soddisfano alle equa- 
zioni lineari $(4)^{\prime \prime \prime}$ (supposte naturalmente risolubili) (dove alle $x_{i}$ si sostituiscano le coordinate $\left(x_{i}{ }^{\circ}\right)$ del punto $A$ ) e, se vogliamo restringerci a spazii reali, anche alle solite disuguaglianze, se questo è possibile. Sia ora $B$ un punto qualunque dello spazio, in cui si vogliono trovare $i$ valori delle $a_{i k}$. Siccome il gruppo opera transitivamente nello spazio ambiente esiste almeno una trasformazione $T$ del gruppo che trasporta il punto $B$ nel punto $A$; essa porterà anche un intorno qualunque di $B$ in un intorno di $A$; di più essa si potrà ottenere con la sola risoluzione di equazioni finite. Sieno $x_{i}^{(1)}$ le coordinate di $B$, e siano $a_{i k} \mathrm{j}$ valori finora incogniti delle $a_{i k}$ nel punto $B$. La $T$, stabilendo una corrispondenza biunivoca tra gli intorni di $B$ o di $A$, stabilisce una corrispondenza biunivoca tra $\mathrm{i}$ punti $x_{i}^{(1)}+d x_{i}^{11}$ e i punti $x_{i}{ }^{\prime)}+d x_{i}^{\prime \prime}$, corrispondenza determinabile con differenziazioni; le $d x_{i}^{\prime{ }^{\prime}}$ vengono date come espressioni lineari omogenee nelle $d x_{i}^{11}$, a coefficienti costanti. Sostituiamo nella $a_{i k}^{(0)} d x_{i}^{(0)} d x_{k}^{(0)}$ alle $d x_{i}^{(0 ;}$ i loro valori così determinati, otterremo una forma quadratica nelle $d x_{i}^{(t)}$, che deve essere identica con $\Sigma a_{i k}^{(1)} d x_{i}^{(1)} d x_{k}^{(1)}$; esprimendo questa identità, si hanno senz'altro i valori delle $a_{i k}^{(1)}$ nel punto qualunque $x_{i}{ }^{13}$ dello spazio ambiente, espressi in funzione delle costanti iniziali $a_{i k}^{(0)}$.

Passiamo ora al caso che il numero $n+m$ delle dimensioni dello spazio sia maggiore del numero $n$ delle variabili $\left(x_{1}, x_{2}, \ldots x_{n}\right)$, su cui opera effettivamente il gruppo, cosicchè nello spazio in discorso il gruppo non opera più transitivamente. $\mathrm{E}$ sia

$$
d s^{2}=\sum_{i, k} \alpha_{i k} d x_{i} d x_{k} \quad(i, k=1,2, \ldots, n+m)
$$

l'elemento lineare dello spazio. Per le $a_{i k}(i, k=n+1, \ldots, n+m)$ nulla vi sarebbe da dire, poichè noi sappiamo già che esse sono funzioni (del resto arbitrarie) delle $x_{n+1}, x_{n+2}, \ldots, x_{n+m}(\S 4)$. In ogni modo si osservi che affinchè il nostro spazio ammetta effettivamente il gruppo in discorso, è condizione necessaria e sufficiente che una trasformazione $T$ del gruppo che porta un punto $B \equiv x_{i}{ }^{1}$ di una varietà $V_{n}$ invariante in un altro punto $A \equiv x_{i}^{(0}$, della stessa $V_{n}$ e quindi stabilisce anche una corrispondenza biunivoca tra gli intorni dei due punti, porti anche la forma

nella forma

$$
\sum_{i, k} a_{i k}\left(x_{i}^{(1)}\right) d x_{i}^{(1)} d x_{k}^{(1)}
$$

$$
\sum_{i, k} a_{i k}\left(x_{l}^{(0)}\right) d x_{i}^{(0 ;} d x_{k}^{0}
$$


Allora prendiamo una varietà qualsiasi $V_{m}$ a $m$ dimensioni che abbia uno e un sol punto comune con ciascuna delle varietà $V_{n}$ minime invarianti e fissiamo in ciascun punto della $V_{m}$ dei valori arbitrarii per le $a_{i k}$ ancora da determinarsi, purchè naturalmente essi soddisfino alle solite equazioni lineari : ciò che corrisponde all'introduzione di funzioni arbitrarie. Per poi determinare $\mathrm{i}$ valori $a_{i k}^{(1)}$ delle $a_{i k}$ in un altro punto $B$ qualsiasi di $S_{n+m}$ basta trovare quel punto $A$, in cui la varietà invariante $V_{n}$ passante per $B$ incontra la $V_{m}$, e in cui le $a_{i k}$ ricevono dei valori noti $a_{i k}^{(0)}$ e applicare quindi ai punti $A, B$ il precedente procedimento. Il nostro teorema è così dimostrato in generale.

Naturalmente non con ogni speciale scelta delle funzioni e costanti arbitrarie su descritte si otterrà un tipo di spazio realmente distinto: anzi per le considerazioni generali del $\S 1$, noi possiamo supporre, senza diminuire la generalità

$$
\begin{array}{lll}
a_{n+1, k}=0 & (k=1,2, \ldots, n, n+2, \ldots, n+m) \\
\left(a_{n+2, k}=0\right)_{x_{n+1}=0} & (k=1=n+2) & a_{n+1, n+1}=1 \\
\left(a_{n+3, k}=0\right)_{x_{n+1}=x_{n+2}=0} & (k=1=n+3) & \left(a_{n+2, n+2}\right)_{x_{n+1}=0}=1 \\
& & \left(a_{n+3, n+3}\right)_{x_{n+1}=x_{n+2}=0}=1, \text { ecc. }
\end{array}
$$

dove le uguaglianze scritte in basso fuori delle parentesi indicano su quali varietà si possono supporre senz'altro verificate le equazioni stesse.

$\S 6$. Ora noi ci vogliamo chiedere qual'è il significato delle relazioni lineari $(4)^{\prime \prime \prime}$, a cui $\mathrm{i}$ valori iniziali delle $a_{i k}$ devono sempre soddisfare. A questa domanda risponde subito la seguente considerazione. Se noi ci riferiamo p. es. al primo dei casi testè trattati e ricordiamo il metodo di cui noi ci siamo serviti, notiamo tosto che per trovare $\mathrm{i}$ valori delle $a_{i h}$ in un punto $B$ in funzione dei valori delle $a_{i k}$ in un punto $A$, ci siamo serviti di una trasformazione $T$ che conducesse il punto $B$ nel punto $A$. Ora nel caso di trasformazioni infinitesime linearmente dipendenti osserviamo che i punti $A, B$ non individuano la trasformazione $T$, ma che anzi di tali trasformazioni ve ne sarà, in generale più di una: chè se $S$ è la trasformazione più generale che lascia fisso $A$, tutte le trasformazioni $T S(0 \quad S T$ a seconda della notazione che si usa) conducono il punto $B$ nel punto $A$. E poichè le $(4)^{\prime \prime \prime}$ ci danno le uniche condizioni a cui devono soddisfare $\mathrm{i}$ valori iniziali delle $a_{i k}$, è ben chiaro che il loro significato è questo, che cioè, qual- 
siasi trasformazione $T S$ venga usata, i valori delle $a_{i k}$ nel punto $B$ siano sempre quelli che si otterrebbero considerando la $T$. In altre parole esse significano, che ogni trasformazione $S$ del gruppo che lascia fisso il punto $A$ deve trasformare in sè la forma $\Sigma a_{i k}^{(0)} d x_{i}^{(0)} d x_{k}^{(0)}$. Del resto risulta senz' altro chiaro dal procedimento usato testè che se questa condizione è soddisfatta, allora $i$ valori che si ottengono in un punto $B$ sono perfettamente determinati, e il gruppo è ammesso da uno spazio, il cui elemento lineare ha dei coefficienti, che nel punto $A$ assumono il valore $a_{i k}^{(0)}$. Possiamo dunque esprimere sotto forma più elegante e concisa le condizioni necessarie e sufficienti affinchè un gruppo possa essere considerato come gruppo di movimenti.

Condizione necessaria e sufficiente affinchè un gruppo $G$ ridotto o no sotto forma normale si possa consideiare come gruppo di movimenti $\dot{e}$ che quel suo sottogruppo $\Gamma$ che lascia fisso un punto $A$ determinato, in cui il gruppo sia regolare, sia tale che esista almeno un sistema di quadriche omotetiche in guisa che un punto infinitamente vicino ad $A$ non esca mai per le trasformazioni di $\Gamma$ da quella di queste quadriche, che passa per esso, o, in altre parole $\Gamma$ trasformi in sè ciascuna quadrica di un sistema di quadriche omotetiche infinitamente vicine ad $A\left({ }^{*}\right)$.

Se il gruppo ̀̀ transitivo basterà chiaramente che questo avvenga in un solo punto (perchè allora avviene in tutti); se è intransitivo ciò dovrà accadere in un punto di ciascuna varietà minima invariante. Questa distinzione è evidentemente superflua, se il gruppo è già ridotto a forma normale.

Se queste condizioni sono soddisfatte, esiste infatti una forma $\sum a_{i k}^{(0} d x_{i}^{(0)} d x_{k}^{(0)}$, che $\Gamma$ trasforma in sè.

Osservazione 1." Se uno di questi cosifatti sistemi di quadriche è formato di elissoidi, lo spazio corrispondente si pù̀ supporre reale.

Osservazione 2. ${ }^{a}$ Le condizioni analitiche, che traducono queste condizioni geometriche sono date dall'annullarsi di quella matrice, che esprime essere le (4) "' compatibili.

$\S 7$. E ben evidente ora che i gruppi $G_{n}$ transitivi su $n$ variabili possono essere considerati come gruppi di movimenti (teorema del prof. Biavchl) perchè in tal caso il sottogruppo $\Gamma$ si riduce all'identità; o in altre parole, è compiutamente determinata la trasformazione che porta un punto $B$ in un punto $A$; e quindi, dati arbitrariamente i valori delle $\alpha_{i k}$ in $A$ riescono senz'altro, coi nostri metodi, determinati i valori delle $a_{i k}$ nel punto $B$. Quest'osservazione rende evidente a priori il bel teorema del prof. Branchr.

$\left.{ }^{*}\right)$ Si noti che così immaginiamo il gruppo operante in uno spazio euclideo. 
Ma questa stessa osservazione si può senz'altro applicare a ogni gruppo con trasformazioni infinitesime linearmente indipendenti e ne traggiamo il teorema :

Ogni gruppo a trasformazioni infinitesime linearmente indipendenti si può considerare come gruppo di movimenti.

Confrontando questo risultato, che è immediato corollario del precedente teorema generale, con quello del $\S 2$ otteniamo il seguente teorema:

Ogni gruppo $G_{n}$ generato da $n$ trasformazioni linearmente indipendenti su un numero qualsiasi $n+m$ di variabili è simile a un gruppo $G_{n}^{\prime}$ semplicemente transitivo in uno spazio ad $n$ dimensioni, e, ciò ch'è lo stesso, si può pensare sottogruppo di un gruppo transitivo su $n+m+k$ lettere e con $n+m+k$ parametri (dove $k$ è un intero qualsiusi nullo o positivo).

Nei precedenti paragrafi noi abbiamo risoluto due questioni: la prima, di riconoscere se un gruppo continuo si può considerare come gruppo di movimenti, la seconda di indicare un mezzo, col quale si possa determinare con sole quadrature, differenziazioni e risoluzione di equazioni finite tutti questi gruppi, e gli spazii che loro corrispondono.

$\S 8$. Noi aggiungeremo ora una facile osservazione, che ci condurrà a un notevole risultato:

Nessun gruppo, che si possa considerare come gruppo di movimenti, è più di una volta transitivo.

Infatti se $G$ è un tale gruppo ed $S$ lo spazio corrispondente, due punti di $S$ hanno almeno un invariante : la loro distanza geodetica.

Per la stessa ragione:

Se un gruppo $G$, si pud considerare come gruppo di movimenti, quel sui sottogruppo che lascia fisso un punto generico è certo intransitivo.

Ne deduciamo:

Nessun spazio $S_{n}$ a un numero $n>2$ di dimensioni pud ammettere un gruppo reale a $\frac{n^{2}+n-2}{2}$ parametri nè come gruppo di movimenti, nè come sottogruppo del gruppo totale di movimenti.

Questo teorema per $n=3$ fu dimostrato dal prof. Bianchi; noi lo ammetteremo vero per $n=m-1$ lo dimostreremo per $n=m$. Sia, se è possi-

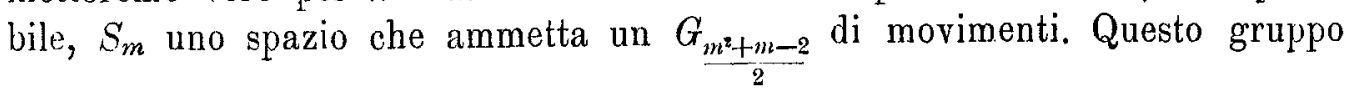
è certamente transitivo, perchè se non lo fosse, esso sarebbe per il teorema del $\S 1$ simile a un gruppo di movimenti di uno spazio a meno di $m$ di- 
mensioni: ciò che è assurdo, perchè $\frac{m^{2}+m-2}{2}>\frac{m(m-1)}{2} \cdot$ Quindi quel suo sottogruppo che lascia fisso un punto generico è proprio un gruppo a

$$
\frac{m^{2}+m-2}{2}-m
$$

parametri, cioè è proprio un $\frac{G_{(m-1)^{*}+(m-1)-2}}{2}$. Questo gruppo per l'osservazione precedente è intransitivo in $G_{n \imath}$ e per il teorema del $\S 1$ è simile a un gruppo di movimenti di uno spazio a $" m-1$ " dimnensioni. Ciò che è pure assurdo, perchè noi abbiamo supposto dimostrato il nostro teorema per $n=m-1$.

La nostra asserzione resta così dimostrata in generale. Così pure si di-

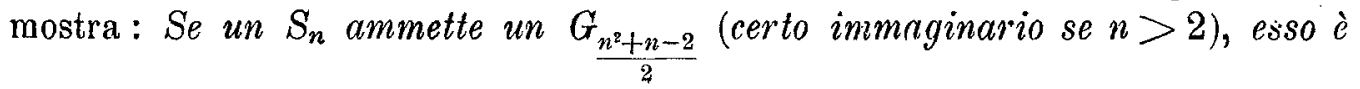

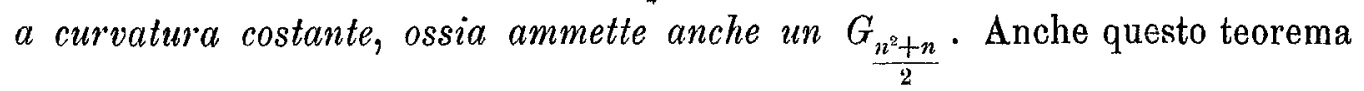
si dimostra col metodo di induzione completa. Ecco p. es. come si dimostra per un $S_{4}$. Quel sottogruppo $G_{5}$ di $G_{3}$ che lascia fisso un punto generico di $S_{4}$ sarà intransitivo e avrà delle $V_{3}$ per varietà minime invarianti, p. es. le $x_{4}=$ cost., geodeticamente parallele. Esso si potrà immaginare operante (transitivamente) sulle $x_{1}, x_{2}, x_{3}(\S 1)$. Questo $G_{5}$ può chiaramente portare ogni segmento $T$ di una di queste varietà in ogni altro segmento della varietà stessa uguale a $T$. Quindi dovendo esso ricondurre tutto lo $S_{4}$ in sè senza mutare le distanze, a due segmenti uguali di una delle $x_{4}=$ cost. cor rispondono (per mezzo delle geodetiche normali alle $x_{4}=$ cost.) due segmenti tra di loro uguali su ogni $x_{4}=$ cost. Ma una $x_{4}=$ cost. ammettendo un $G_{5}$, ammetterà un $G_{6}$. Quel gruppo $G_{6}^{\prime}$ che opera sulle $x_{1}, x_{2}, x_{3}$ come questo $G_{6}$ e lascia invariato $x_{4}$ trasformerà dunque in sè ogni $x_{1}=$ cost., senza mutar l'elemento lineare e perciò sarà un gruppo di movimenti dello $S_{4}$, che quindi ammetterà un $G_{10}$.

$\S 9$. Ritorniamo ora alle condizioni necessarie e sufficienti date al $\S 6$ affinchè un dato gruppo continuo si possa considerare come un gruppo di movimenti. Queste condizioni si possono anche enunciare cos̀̀: Quel sottogruppo che lascia fisso un punto $A$ deve essere un sottogruppo di uno spazio a curvatura costante, quando lo si pensi operante sulle rette uscenti da $A$, considerate come elementi, o, in altre parole, deve lasciare fisso un cono quadrico col vertice in $A$; di più egli deve lasciare invariate le quadriche in- 
finitamente vicine ad $A$ aventi questo cono per cono assintotico. La prima parte di queste condizioni non sarebbe evidentemente sufficiente, perchè p. es. anche il $G_{7}$ delle similitudini dello spazio euclideo soddisfa ad esse. Esistono dunque altri gruppi che soddisfano soltanto a quella prima condizione e che non sono altro che i gruppi di trasformazioni conformi. Osserverò che la condizione affinchè una trasformazione infinitesima generi un gruppo di trasformazioni conformi per lo spazio

$$
d s^{2}=\Sigma a_{i k} d x_{i} d x_{k}
$$

è che esista una funzione $k$ delle $x_{i}$ tale che

$$
X\left(\Sigma a_{i k} d x_{i} d x_{k}\right)=k \Sigma a_{i k} d x_{i} d x_{k} \text {. }
$$

Se $k$ è costante essa si può chiamare una trasformazione simile.

Un'altra osservazione è la seguente: Almeno per gruppi transitivi valgono ancora i teoremi seguenti, che si dimostrano come gli analoghi dei paragrafi precedenti per le forme quadratiche:

Si possono costruire per quadrature tutte le forme differenziali di ordine qualunque che ammettono un gruppo continuo transitivo di operazioni, e $i$ gruppi corrispondenti.

Condizione necessaria e sufficiente affinchè un gruppo transitivo possa trasformare in sè una forma differenziale di ordine qualunque $n$, ̀̀ che quel suo sottogruppo che lascia fisso un punto A trasformi $i$ punti infinitamente vicini ad $A$ in modo che esista un sistema di superficie omotetiche di nesimo ordine infinitamente vicine ad $A$, ciascuna delle quali venga dal detto sottogruppo trasformata in sè.

Così si possono pure generalizzare molti altri dei precedenti risultati.

$\S 10$. Ricerchiamo ora effettivamente i gruppi a 1, 2, 3, 4 parametri che possono essere realmente considerati come gruppi di movimenti. Osserviamo che se il numero delle loro trasformazioni dipendenti è minore o uguale a 3 , essi devono ( $\$ 1$ ) potersi considerare come gruppi di movimenti di uno spazio a non più di tre dimensioni, e quindi si possono prendere senz'altro dalla Memoria del prof. Bianchi; si potrebbe però anche, poichè il Liw diede tutti i gruppi su due variabili e insegnò un metodo per trovare tutti queili su tre variabili, esaminare l'un dopo l'altro tutti quelli dei tipi enunciati da LIE che non posseggono più di quattro parametri, e di questi ritenere soltanto quelli che soddisfano alle condizioni del $\S 6$. Questo metodo sarebbe assai rapido, e servirebbe a trovare senz'altro direttamente $i$ ri- 
sultati della più volte citata Memoria del prof. Bianchi. Si potrebbe anche infine costruire questi gruppi secondo il mio metodo del $\S 5$; ma questa via sarebbe un po' più lunga della precedente. Noi naturalmente prenderemo questi gruppi addirittura dalla Memoria del prof. Branchi, perchè il lettore può facilmente da sè vedere che questi altri metodi condurrebbero appunto ai risultati del prof. Branchr.

\section{Gruppi $G_{1}$ :}

$$
X_{1}=\frac{\partial}{\partial x_{1}} .
$$

Gruppi $G_{2}$ a trasformazioni infinitesime naturalmente linearmente indipendenti.

$$
\begin{aligned}
\left.\mathrm{I}^{\circ}\right) & X_{1}=\frac{\partial}{\partial x_{1}} & X_{2}=\frac{\partial}{\partial x_{2}} \\
\left.\mathrm{II}^{\circ}\right) & X_{1}=e^{-x_{2}} \frac{\partial}{\partial x_{1}} & X_{2}=\frac{\partial}{\partial x_{2}} .
\end{aligned}
$$

Gruppi $G_{3}$ a trasformazioni infinitesime linearmente dipendenti.

$$
\begin{aligned}
\left.\mathrm{I}^{\mathrm{O}}\right) \quad X_{1}=\frac{\partial}{\partial x_{1}} \quad X_{2}=\frac{\partial}{\partial x_{2}} \quad X_{3}=x_{2} \frac{\partial}{\partial x_{2}}-x_{1} \frac{\partial}{\partial x_{2}} \\
\left.\mathrm{II}^{\circ}\right) \quad X_{1}=\frac{\partial}{\partial x_{2}} \quad X_{2}=\operatorname{sen} x_{2} \frac{\partial}{\partial x_{1}}+\operatorname{cotg} x_{1} \cos x_{2} \frac{\partial}{\partial x_{2}} \quad X_{3}=\left(X_{1} X_{2}\right) \\
\left.\mathrm{III}^{\circ}\right) \quad X_{1}=\frac{\partial}{\partial x_{2}} \quad X_{2}=\frac{\partial}{\partial x_{1}}-x_{2} \frac{\partial}{\partial x_{2}} \quad X_{3}=x_{2} \frac{\partial}{\partial x_{1}}+\frac{1}{2}\left(e^{-2 x_{1}}-x_{2}^{2}\right) \frac{\partial}{\partial x_{2}} .
\end{aligned}
$$

Gruppi $G_{3}$ a trasformazioni infinitesime linearmente indipendenti.

$$
\begin{aligned}
&\left.I^{\prime \prime}\right) \quad X_{1}=\frac{\partial}{\partial x_{2}} ; \quad X_{2}=\frac{\partial}{\partial x_{3}} ; \quad X_{3}=-\frac{\partial}{\partial x_{1}}+\left(\frac{x_{2}}{2}+x_{3}\right) \frac{\partial}{\partial x_{2}}+\frac{x_{3}}{2} \frac{\partial}{\partial x_{3}} \\
&\text { II } \left.^{\circ}\right) \quad X_{1}=\frac{\partial}{\partial x_{2}} ; \quad X_{2}=\frac{\partial}{\partial x_{3}} ; \quad X_{3}=-\frac{\partial}{\partial x_{1}}+x_{2} \frac{\partial}{\partial x_{2}}+h x_{3} \frac{\partial}{\partial x_{3}} \\
&\text { III } \left.^{\circ}\right) \quad X_{1}=\frac{\partial}{\partial x_{2}} ; \quad X_{2}=\frac{\partial}{\partial x_{3}} ; \quad X_{3}=\frac{\partial}{\partial x_{1}}-x_{3} \frac{\partial}{\partial x_{2}}+\left(x_{2}+h x_{3}\right) \frac{\partial}{\partial x_{3}} \\
&\text { IV } \left.^{\circ}\right) \quad X_{1}=e^{x_{3}} \frac{\partial}{\partial x_{1}}-x_{2}^{2} e^{x_{3}} \frac{\partial}{\partial x_{2}}-2 x_{2} e^{-x_{3}} \frac{\partial}{\partial x_{3}} ; \quad X_{2}=\frac{\partial}{\partial x_{3}} ; \quad X_{3}=e^{x_{1}} \frac{\partial}{\partial x_{3}} \\
&\text { V } \left.^{\circ}\right) \quad X_{1}=\frac{\partial}{\partial x_{2}} ; \quad X_{2}=\cos x_{2} \frac{\partial}{\partial x_{1}}-\operatorname{cotg} x_{1} \operatorname{sen} x_{3} \frac{\partial}{\partial x_{2}}+\frac{\operatorname{sen} x_{2}}{\operatorname{sen} x_{1}} \frac{\partial}{\partial x_{3}} ; \\
& X_{3}=\left(X_{1} X_{2}\right)
\end{aligned}
$$


$\left.\mathrm{VI}^{0}\right) \quad X_{1}=\frac{\partial}{\partial x_{2}} ; \quad X_{2}=\frac{\partial}{\partial x_{3}} ; \quad X_{3}=-\frac{\partial}{\partial x_{1}}+x_{3} \frac{\partial}{\partial x_{2}}$

$\left.\mathrm{VII}^{\circ}\right) \quad X_{1}=\frac{\partial}{\partial x_{1}} ; \quad X_{2}=\frac{\partial}{\partial x_{2}} ; \quad X_{3}=\frac{\partial}{\partial x_{3}}$.

Gruppi $G_{4}$ a trasformazioni infinitesime linearmente dipendenti.

$\left.I^{0}\right) \quad X_{1}=\frac{\partial}{\partial x_{2}} ; \quad X_{2}=\frac{\partial}{\partial x_{3}} ; \quad X_{3}=-\frac{\partial}{\partial x_{1}}+x_{3} \frac{\partial}{\partial x_{2}} ;$

$$
X_{4}=x_{3} \frac{\partial}{\partial x_{1}}+\frac{1}{2}\left(x_{1}^{\prime}-x_{3}^{2}\right) \frac{\partial}{\partial x_{2}}-x_{1} \frac{\partial}{\partial x_{3}}
$$

$\left.\mathrm{II}^{\prime \prime}\right) \quad X_{1}=\frac{\partial}{\partial x_{2}} ; \quad X_{3}=\frac{\partial}{\partial r_{3}} ; \quad X_{3}=\frac{\partial}{\partial x_{1}}-x_{2} \frac{\partial}{\partial x_{2}}$;

$$
X_{4}=x_{2} \frac{\partial}{\partial x_{1}}+\frac{1}{2}\left(\frac{e^{-2 x_{1}}}{1-n^{2}}-x_{2}^{\prime}\right) \frac{\partial}{\partial x_{2}}-\frac{n e^{-x_{1}}}{1-n^{2}} \frac{\partial}{\partial} \bar{x}_{3}
$$

$\left.\mathrm{III}^{\circ}\right) \quad X_{1}=\frac{\partial}{\partial x_{2}} ; \quad X_{2}=\frac{\partial}{\partial x_{3}} ;$

$$
X_{3}=\cos x_{2}-\operatorname{cotg} x_{1} \operatorname{sen} x_{2} \frac{\partial}{\partial x_{z}}+\frac{n \operatorname{sen} x_{2}}{\operatorname{sen} x_{1}} \frac{\partial}{\partial x_{3}} ; \quad X_{4}=\left(X_{1} X_{3}\right) .
$$

Il tipo $\mathrm{II}^{\circ}$ ) e $\left.\mathrm{III}\right)^{\circ}$ si potrebbero da un punto di vista generale considerare come identici.

$\S$ 11. Troviamo ora i gruppi $G_{4}$ a trasformazioni infinitesime indipendenti. Questo gruppo $(\S 1)$ si deve poter ridurre a un gruppo transitivo su 4 lettere. Per determinare questi gruppi potremmo, seguendo il procedimento generale del $\S 5$, ricorrere senz'altro ai metodi di LiE. Ma però noi possiamo usare di metodi più rapidi, in quanto che noi conosciamo tulti i $G_{3}$ transitivi. Potremo dunque prendere un sottogruppo $G_{3}$ di $G_{4}$, di cui cercheremo la composizione. Da questa potremo subito dedurre (per mezzo della penultima tabella) una forma, a cui possiamo immaginare ridotte le sue trasformazioni infinitesime; la quarta trasformazione infinitesima di $G_{4}$ si determina poi in guisa che non sia combinazione lineare dello precedenti, e che il $G_{1}$ abbia la composizione voluta.

Sia ora il $G_{4}$ non integrabile (cfr. Lie: Vol. III, $\S 137$ ); il gruppo derivato $\left(X_{1}, X_{2}, X_{3}\right)$ dovrà avere una delle seguenti composizioni:

oppure

$$
\left(X_{1} X_{2}\right)=X_{1} \quad\left(X_{1} X_{3}\right)=2 X_{2} \quad\left(X_{2} X_{3}\right)=X_{3}
$$

$$
\left(X_{1} X_{2}\right)=X_{3} \quad\left(X_{2} X_{3}\right)=X_{1} \quad\left(X_{3} X_{1}\right)=X_{1} .
$$


$\mathrm{E}$ in ambi i casi si può poi scegliere $X_{4}$ in modo che sia permutabile con le precedenti trasformazioni.

Il $G_{3}\left(X_{1}, X_{2}, X_{3}\right)$ è certo a trasformazioni infinitesime linearmente indipendenti e (§ 1) si può immaginare semplicemente transitivo su tre lettere.

$\mathrm{Nel}$ primo di questi casi avremo:

$$
X_{1}=e^{-x_{3}} \frac{\partial}{\partial x_{1}}-x_{2}^{2} e^{-x_{3}} \frac{\partial}{\partial x_{2}}-2 x_{2} e^{-x_{3}} \frac{\partial}{\partial x_{3}} ; \quad X_{2}=\frac{\partial}{\partial x_{3}} ; \quad X_{3}=e^{x_{3}} \frac{\partial}{\partial x_{2}} .
$$

Posto :

$$
X_{4}=\sum_{1}^{4} \xi_{i} \frac{\partial}{\partial x_{i}} \quad\left(\xi_{i}=\xi_{i}\left(x_{1}, x_{2}, x_{3}, x_{4}\right)\right)
$$

avremo, essendo $\left(X_{i}, X_{4}\right)=0(i=1,2,3)$

$$
\begin{aligned}
& e^{-x_{3}} \frac{\partial \xi_{1}}{\partial x_{1}}+\xi_{3} e^{-x_{3}}=0 ; \quad e^{-x_{3}} \frac{\partial \xi_{2}}{\partial x_{1}}+2 x_{2} \xi_{2} e^{-x_{3}}-\xi_{3} e^{-\left(x_{3}+x_{3}\right)}=x_{2}^{2} e^{-x_{3} \xi_{3}} \\
& e^{-x_{3}} \frac{\partial \xi_{3}}{\partial x_{1}}+2 \xi_{2} e^{-x_{3}}-2 \xi_{3} x_{2} e^{-x_{3}}=0 \\
& e^{-x x_{3}} \frac{\partial \xi_{4}}{\partial x_{1}}=\frac{\partial \xi_{1}}{\partial x_{2}}=\frac{\partial \xi_{3}}{\partial x_{2}}=\frac{\partial \xi_{4}}{\partial x_{2}}=0 \quad \frac{\partial \xi_{2}}{\partial x_{2}}-\xi_{3}=0 \quad \frac{\partial \xi_{i}}{\partial x_{3}}=0(i=1,2,3)
\end{aligned}
$$

donde, integrando,

$$
\begin{array}{ll}
\xi_{1}=\alpha x_{1}^{2}+2 \beta x_{1}+\gamma \quad \xi_{2}=-2 x_{2}\left(\alpha x_{1}+\beta\right)+\alpha \\
\xi_{3}=-2\left(\alpha x_{1}+\beta\right) & \xi_{1}=-\frac{1}{l_{1}}
\end{array}
$$

dove $\alpha, \beta, \gamma, l_{4}$ sono funzioni qualunque di $x_{4}$.

Con procedimento analogo troviamo nel secondo caso:

$$
X_{1}=\frac{\partial}{\partial x_{2}} ; \quad X_{2}=\cos x_{2} \frac{\partial}{\partial x_{1}}-\operatorname{cotg} x_{1} \operatorname{sen} x_{2} \frac{\partial}{\partial x_{2}}+\frac{\operatorname{sen} x_{2}}{\operatorname{sen} x_{1}} \frac{\partial}{\partial x_{3}} \quad X_{3}=\left(X_{1} X_{2}\right) .
$$

E, posto

si ottiene

$$
X_{4}==\sum_{i=1}^{4} \xi_{i}\left(x_{1}, x_{2}, x_{3}, x_{4}\right) \frac{\partial}{\partial x_{i}}
$$

$$
\xi_{1}=\xi_{2}=0 \quad X_{1}=\varphi \frac{\partial}{\partial x_{3}}+\psi \frac{\partial}{\partial x_{4}}
$$

dore $\varphi, \psi$ sono funzioni di $x_{\downarrow}$. 
Del resto questi due casi, quando non si distingua reale da immaginario, sono da riputarsi identici.

Sia ora il $G_{4}$ integrabile e sia un $G_{3}$ a trasformazioni non permutabili il giruppo derivato. Indicando con $X_{1}, X_{2}, X_{3}, X_{4}$ le trasformazioni generatrici di $G_{4}$ potremo porre (Lie, loc. cit.) o

$\left.\mathrm{I}^{\circ}\right)$

$$
\begin{aligned}
& \left(Y_{1} X_{2}\right)=\left(X_{1} X_{3}\right)=0 \quad\left(X_{2} X_{3}\right)=X_{1} \\
& \left(X_{1} X_{4}\right)=c X_{1} \quad\left(X_{2} Y_{4}\right)=X_{2} \quad\left(X_{3} X_{4}\right)=(c-1) X_{3}
\end{aligned}
$$

oppure

$\left.\mathrm{II}^{\circ}\right)$

$$
\begin{aligned}
& \left(X_{1}, X_{2}\right)=\left(X_{1} X_{3}\right)=0 \quad\left(X_{2} X_{3}\right)=X_{1} \\
& \left(X_{1} Y_{4}\right)=2 X_{1} ; \quad\left(X_{2} X_{4}\right)=X_{2} ; \quad\left(X_{3} X_{4}\right)=X_{2}+X_{3} \text {. }
\end{aligned}
$$

In $a \mathrm{mbi} \cdot \mathrm{i}$ casi potremo fare

$$
X_{1}=\frac{\partial}{\partial x_{2}} ; \quad X_{2}=\frac{\partial}{\partial x_{3}} ; \quad X_{3}=-\frac{\partial}{\partial x_{1}}+x_{3} \frac{\partial}{\partial x_{2}} .
$$

E posto

$$
X_{4}=\sum_{i=1}^{4} \xi_{i} \frac{\partial}{\partial x_{i}}
$$

avremo, nel primo caso,

$$
\begin{array}{ll}
\xi_{1}=(c-1) x_{1}+\xi_{1} & \xi_{2}=-x_{1} \varphi_{3}+c x_{2}+\varphi_{2} \\
\xi_{3}=x_{3}+\varphi_{3} & \xi_{4}=-\frac{1}{l_{4}}
\end{array}
$$

e nel secondo

$$
\begin{array}{ll}
\xi_{1}=x_{1}+\varphi_{1} & \xi_{2}=-\frac{x_{1}^{2}}{2}-x_{1} \xi_{3}+2 x_{2}+\varphi_{2} \\
\xi_{3}=x_{3}-x_{1}+\varphi_{3} & \xi_{4}=-\frac{1}{l_{4}}
\end{array}
$$

dove $\varphi_{1}, \varphi_{2}, \varphi_{3}, l_{4}$ sono funzioni di $x_{4}$.

Sia ora il gruppo derivato di $G_{4}$ un $G_{2}$ e non contenga il $G_{4}$ un $G_{3}$ a trasformazioni permutabili. Allora 0 il $G_{4}$ ha la penultima composizione, dove si ponga $c=1$, oppure avrà la composizione:

$$
\left(X_{1} X_{2}\right)=\left(X_{2} X_{3}\right)=\left(\begin{array}{ll}
X_{1} X_{4}
\end{array}\right)=\left(\begin{array}{ll}
X_{3} X_{4}
\end{array}\right)=0 \quad\left(X_{1} X_{3}\right)=X_{1} \quad\left(X_{2} X_{4}\right)=X_{2} .
$$

Potremo fare:

$$
X_{1}=\frac{\partial}{\partial x_{2}} ; \quad X_{2}=\frac{\partial}{\partial x_{3}} ; \quad X_{3}=-\frac{\partial}{\partial x_{1}}+x_{2} \frac{\partial}{\partial x_{2}}
$$


e, posto

$$
X_{4}=\sum_{i=1}^{4} \xi_{i} \frac{\partial}{\partial x_{i}}
$$

avremo:

$$
\xi_{1}=\varphi_{1} \quad \xi_{2}=e^{-x_{i}} \varphi_{2} \quad \xi_{3}=x_{3}+\varphi_{3} \quad \xi_{4}=-\frac{1}{l_{4}},
$$

dove $\varphi_{1}, \varphi_{2}, \rho_{3}, l_{4}$ sono funzioni di $x_{4}$.

Contenga ora il $G_{4}$ un $G_{3} \equiv\left(X_{1}, X_{2}, X_{3}\right)$ a trastormazioni a due a due permutabili. Potremo fare

$$
X_{1}=\frac{\partial}{\partial x_{1}}, \quad X_{2}=\frac{\partial}{\partial x_{2}}, \quad X_{3}=\frac{\partial}{\partial x_{3}}, \quad X_{4}=\sum_{i=1}^{4} \xi_{i} \frac{\partial}{\partial x_{i}} .
$$

Si potrà poi scegliere $X_{4}$ in guisa che o

$$
\left.I^{0}\right) \quad\left(X_{1} X_{4}\right)=X_{1} \quad\left(X_{2} X_{4}\right)=a X_{2} \quad\left(X_{3} X_{4}\right)=c X_{3}
$$

e quindi

$$
\xi_{1}=x_{1}+\varphi_{1}, \quad \xi_{2}=a x_{2}+\varphi_{2}, \quad \xi_{3}=c x_{3}+\varphi_{3}, \quad \xi_{4}=-\frac{1}{l_{4}}
$$

oppure

$$
\left.\mathrm{II}^{\circ}\right) \quad\left(X_{1} X_{4}\right)=c X_{1} \quad\left(X_{2} X_{4}\right)=(1+c) X_{2} \quad\left(X_{3} X_{4}\right)=X_{1}+c X_{3}
$$

e quindi :

$$
\xi_{1}=c x_{1}+x_{3}+\varphi_{1} ; \quad \xi_{2}=(1+c) x_{2}+\xi_{2} ; \quad \xi_{3}=c x_{3}+\varphi_{3} ; \quad \xi_{1}=-\frac{1}{l_{1}}
$$

oppure

$$
\text { III } \left.^{\circ}\right) \quad\left(X_{1} X_{4}\right)=X_{2} \quad\left(X_{2} X_{4}\right)=0 \quad\left(X_{3} X_{4}\right)=X_{1}
$$

e quindi :

$$
\xi_{1}=x_{3}+\varphi_{1} \quad \xi_{2}=x_{1}+\varphi_{2} \quad \xi_{3}=x_{3}+\varphi_{3} \quad \xi_{4}=-\frac{1}{l_{4}}
$$

oppure

IV $) \quad\left(X_{1} X_{4}\right)=X_{1}+X_{2} \quad\left(X_{2} X_{4}\right)=X_{2} \quad\left(X_{3} X_{4}\right)=X_{1}+X_{3}$

e quindi :

$$
\xi_{1}=\rho_{1} \quad \xi_{2}=x_{3}+\varphi_{2} \quad \xi_{3}=\varphi_{3} \quad \xi_{4}=-\frac{1}{l_{1}}
$$

oppure :

$$
\left.\nabla^{\circ}\right) \quad\left(X_{1} X_{1}\right)=\left(X_{2} X_{4}\right)=0, \quad\left(X_{3} X_{4}\right)==X_{2}
$$


e quindi :

oppure :

$$
\xi_{1}=\varphi_{1} ; \quad \xi_{2}-x_{3}+\varphi_{2} ; \quad \xi_{3}=\xi_{3} ; \quad \xi_{4}=-\frac{1}{l_{4}}
$$

$\left.\mathrm{VI}^{\circ}\right) \quad\left(X_{1} X_{4}\right)=X_{1} \quad\left(X_{2} X_{4}\right)=X_{2} \quad\left(X_{3} X_{4}\right)=X_{2}+X_{3}$

e quindi :

$$
\xi_{1}=x_{1}+\varphi_{1} \quad \xi_{2}=x_{2}+x_{3}+\varphi_{2} \quad \xi_{3}=x_{3}+\varphi_{3} \quad \xi_{4}=-\frac{1}{l_{4}}
$$

oppure

$\left.\mathrm{VII}{ }^{\circ}\right)$

$$
\left(X_{i} X_{4}\right)=0 \quad(i=1,2,3)
$$

e quindi

$$
X_{4}=\varphi_{1} \frac{\partial}{\partial x_{1}}+\varphi_{2} \frac{\partial}{\partial x_{2}}+\varphi_{3} \frac{\partial}{\partial x_{3}}-\frac{1}{l_{1}} \frac{\partial}{\partial x_{4}}
$$

dove $\varphi_{1}, \varphi_{i}, \varphi_{3}, l_{4}$ sono funzioni di $x_{4}$.

$\S 12$. Veniamo ora a un'altra parto del presente lavoro, dove io voglio esporre un metodo per la ricerca dei sottogruppi finiti discontinui dei gruppi continui e, in modo speciale, dei gruppi che si possono considerare come gruppi di movimenti. Io svolgerò questi metodi, applicandoli dapprima effettivamente a due esempii, agli spazii cioè del tipo VIII ${ }^{\circ}$ ) e $\mathrm{IX}^{\circ}$ ) del prof. Branonr, ciò che ci permetterà anche di trovare delle notevolissime rappresentazioni geometriche di tali spazii sulla sfera e sulla pseudosfera.

Cominceremo dagli spazii del tipo $1 \mathrm{X}^{\circ}$ ), che ammettono, con le notazioni del prof. Bianchi un gruppo generato dalle

$$
\begin{gathered}
X_{1}=\frac{\partial}{\partial x_{2}} ; \quad X_{2}=\cos x_{2} \frac{\partial}{\partial \cdot \overline{x_{1}}}-\operatorname{cotg} x_{1} \operatorname{sen} x_{2} \frac{\partial}{\partial x_{2}}+\frac{\operatorname{sen} x_{2}}{\operatorname{sen} x_{1}} \frac{\partial}{\partial \cdot x_{3}} \\
X_{3}=\left(X_{1} X_{2}\right)
\end{gathered}
$$

con la composizione :

$$
\left(X_{1} X_{2}\right)=X_{3} ; \quad\left(X_{2} X_{3}\right)=X_{1} ; \quad\left(X_{3} X_{1}\right)=X_{2} .
$$

Costruiamo il gruppo aggiunto di questo gruppo. Con le usuali notazioni di Lie, esso si ottiene dall'integraziono del sistema:

$$
\begin{aligned}
& \frac{d e_{1}^{\prime}}{d t}=\lambda_{2} e_{3}^{\prime}-\lambda_{3} e_{2}^{{ }_{2}} \\
& \frac{d e_{2}}{d t}=\lambda_{3} e_{1}-\lambda_{1} e_{3}^{\prime} \\
& \frac{d e_{3}^{\prime}}{d t}=\lambda_{1} e_{2}-\lambda_{2} e_{1}^{\prime_{1} .}
\end{aligned}
$$


Donde

$$
e_{1}^{\prime} \frac{d e_{1}^{\prime}}{d t}+e_{2}^{\prime} \frac{d e_{2}^{\prime}}{d t}+e_{3}^{\prime} \frac{d e_{3}^{\prime}}{d t}=0
$$

ossia

$$
e_{1}^{\prime 2}+e_{2}^{\prime z}+e_{3}^{\prime 2}=\text { cost. }
$$

Questo gruppo aggiunto non è perciò altro che il gruppo delle rotazioni di una sfera in sè slessa; e poichè se $T$ è una trasformazione qualunque del gruppo iniziale

$$
T^{-1} T T=T
$$

ne traggiamo che alla trasformazione $\lambda_{1} t, \lambda_{2} t, \lambda_{3} t$ del gruppo iniziale corrisponde una rotazione della sfera attorno al diametro che ha $i$ coseni di direzioni proporzionali a $\lambda_{1} t, \lambda_{2} t, \lambda_{3} t$; cio che si conferma col calcolo seguente. Derivando la (1) rispetto a $t$, otteniamo per le (2) e (3):

$$
\frac{d^{2} e_{1}^{\prime}}{d t^{2}}+\left(\lambda_{2}^{0}+\lambda_{3}^{2}\right) e_{1}^{\prime}-\lambda_{1}\left(\lambda_{2} e_{2}^{\prime}+\lambda_{3} e_{3}^{\prime}\right)=0
$$

donde :

$$
\frac{d^{3} e_{1}^{\prime}}{d t^{3}}+\left(\lambda_{2}^{2}+\lambda_{3}^{2}\right) \frac{d e_{1}^{\prime}}{d t}-\lambda_{1}\left(\lambda_{2} \frac{d e_{3}^{\prime}}{d t}+\lambda_{3} \frac{d e_{3}^{\prime}}{d t}\right)=0
$$

e poichè per le (1), (2), (3) si ha

si avrà

$$
\sum_{i}^{3} \lambda_{i} \frac{d e^{\prime} i}{d t}=0
$$

$$
\frac{d^{3} e^{\prime} t}{d t^{3}}+\left(\lambda_{1}^{2}+\lambda_{z}^{2}+\lambda_{3}^{2}\right) \frac{d e_{1}^{\prime}}{d t}=0
$$

Identica equazione si trova per $e_{2}^{\prime}, e^{\prime}{ }_{3}$. Indicando con $A_{i}, B_{i}, C_{i}$ delle costanti avremo dunque

dove

$$
e_{i}^{i}=A_{i}+B_{i} \operatorname{sen} H t+C_{i} \cos H t \quad(i=1,2,3)
$$

$$
H^{2}=\lambda_{1}^{2}+\lambda_{2}^{2}+\lambda_{3}^{2}
$$

Servendoci delle (1), (2), (3) e del fatto che per $t=0$

$$
e_{i}^{\prime}=e_{i} \quad(i=1,2,3)
$$

potremo determinare le $A_{i}, B_{i}, C_{i}$ e otterremo infine, serivendo $\lambda_{1}, \lambda_{2}, \lambda_{3}, H$ 
in luogo di $\lambda_{1} t, \lambda_{2} t, \lambda_{3} t, H t$

$$
\left.\begin{array}{rl}
e_{1}^{\prime}=e_{1}\left(\frac{\lambda_{1}^{3}}{H^{2}}+\cos H\left(1-\frac{\lambda_{1}^{2}}{H^{2}}\right)+e_{2}\left(\frac{\lambda_{1} \lambda_{2}}{H^{2}}+\frac{\lambda_{3}}{H} \operatorname{sen} H-\frac{\lambda_{1} \lambda_{2}}{H^{2}} \cos H\right)\right. \\
+e_{3}\left(\frac{\lambda_{1} \lambda_{3}}{H^{2}}-\frac{\lambda_{2}}{H} \operatorname{sen} H-\frac{\lambda_{1} \lambda_{3}}{H^{2}} \cos H\right)
\end{array}\right\}
$$

e le eguaglianze che se ne ottengono rotando. Queste eguaglianze rappresentano appunto una rotazione attorno alla retta, $\mathrm{i}$ cui coseni di direzione sono proporzionali a $\lambda_{1}, \lambda_{2}, \lambda_{3}$. Per trovarne l'ampiezza $\theta$, si osservi che per note formole di Geometria analitica

$$
\left\|\begin{array}{lll}
\lambda_{1} & \lambda_{2} & \lambda_{3} \\
e_{1} & e_{2} & e_{3}
\end{array}\right\| \cdot\left\|\begin{array}{ccc}
\lambda_{1} & \lambda_{2} & \lambda_{3} \\
e_{1}^{\prime} & e_{2}^{\prime} & e_{3}^{\prime}
\end{array}\right\|=\cos \theta\left\|\begin{array}{lll}
\lambda_{1} & \lambda_{2} & \lambda_{3} \\
e_{1} & e_{2} & e_{3}
\end{array}\right\|^{*}
$$

donde sostituendo e sviluppando otteniamo:

$$
\theta=H \text {. }
$$

Abbiamo così trovato, con metodo evidentemente applicabile a casi più generali, un gruppo di sostituzioni lineari isomorfo al gruppo dato. E il nostro risultato si può esporre così :

Il gruppo in discorso è oloedricamente isomorfo al gruppo dei movimenti di una sfera in sè : l'isumorfismo si stabilisce, facendo corrispondere alla trasformazione

$$
\lambda_{1} X_{1}+\lambda_{2} X_{2}+\lambda_{3} X_{3}
$$

una rotazione di ampiezza $\sqrt{\lambda_{1}^{2}+\lambda_{2}^{2}+\lambda_{2}^{3}}$ attorno a quel punto di una sfera (col centro nell'origine) le cui coordinate sono proporzionali $a \lambda_{1}, \lambda_{2}, \lambda_{3}$.

I gruppi non sono pero simili, perchè mentre uno opera su tre variabili, l'altro opera in realtà soltanto su due.

$\mathrm{Ma}$ ora osserviamo che dati due gruppi oloedricamente isomorfi, non sempre dall'esistenza di sottogruppi finiti discontinui per l'uno si può conchiudere l'esistenza di sottogruppi finiti discontinui per l'altro. Così p. es. il gruppo delle rotazioni attorno a un asse e il gruppo delle traslazioni in una direzione sono oloedricamente isomorfi, mentre però per l'uno esistono sottogruppi discontinui finiti e per l'altro non ne esistono affatto.

Cio che si deve alle polidromie, ecc., che si possono presentare nmpliando il campo in cui opera un dato gruppo. È ben chiaro però che a un sottogruppo finito discontinuo di un gruppo, corrisponderà un sottogruppo 
isomorfo nel gruppo aggiunto, in generale. E quiridi dovremo prima ricercare i sottograppi discontinui finiti del gruppo aggiunto. Questo prołlema è senz'altro risoluto nel nostro caso: i sottogruppi cercati nel gruppo aggiunto non sono che i gruppi dei poliedri regolari. E il nostro calcolo precedente definisce senza ambiguità $\mathrm{i}$ sottogruppi corrispondenti del gruppo iniziale, perchè dà nel modo più perspicuo quale trasformazione del nostro gruppo si deve far corrispondere a una trasformazione del gruppo aggiunto. Ma per poter affermare che le trasformazioni del gruppo iniziale, corrispondenti alle trasformazioni del gruppo di un poliedro regolare contenuto nel gruppo aggiunto, formino effettivamente un gruppo, bisogna, come risulta chiaramente dalle considerazioni precedenti, fissare bene il significato delle coordinate. A tal fine serve l'importante osservazione che due gruppi oloedricamente isomorfi non simili si possono spesso rendere simili, mutando le variabili su cui essi operano. Nel caso nostro il gruppo aggiunto, invece di essere considerato come un gruppo operante sui punti della sfera, sarà pensato come gruppo operante sugli elementi della sfera, ciò̀ sulla sfera immaginata come luogo degli infiniti enti che si definiscono dando un punto della sfera stessa e una direzione uscente da esso tangente alla sfera. I due gruppi risultano allora senz'altro simili, e con opportuna scelta delle coordinate di un elemento della sfera, essi riescono identici. Si prendano come coordinate di un elemento della sfera la colatitudine e la longitudine $\theta, \varphi$ del punto corrispondente e la derivata $\theta(1)=\frac{d \theta}{d s}$, dove $d s$ è la lunghezza dell'elemento, $d \theta$ l'incremento di $\theta$ muovendoci lungo di esso. Le trasformazioni infinitesime del gruppo aggiunto sono

Posto

$$
e_{3} \frac{\partial}{\partial e_{2}}-e_{2} \frac{\partial}{\partial e_{3}} ; \quad e_{1} \frac{\partial}{\partial e_{3}}-e_{3} \frac{\partial}{\partial e_{1}} ; \quad e_{2} \frac{\partial}{\partial e_{1}}-e_{1} \frac{\partial}{\partial e_{z}} \text {. }
$$

$$
e_{3}=\cos \theta, \quad e_{2}=\operatorname{sen} \theta \operatorname{sen} \varphi, \quad e_{1}=\operatorname{sen} \theta \cos \varphi
$$

avremo che l'ultima, rappresentando una rotazione attorno all'asse delle " $e_{3}$ " sarà eguale a

$$
X_{3}=\frac{\partial}{\partial \varphi}
$$

La seconda, essendo una rotazione attorno all' asse $e_{2}$, lascia invariato $\operatorname{sen} \theta \operatorname{sen} \varphi$ e quindi sarà della forma

$$
e_{1} \frac{\partial}{\partial e_{3}}-e_{3} \frac{\partial}{\partial e_{1}}=k\left(-\cos \theta \operatorname{sen} \varphi \frac{\partial}{\partial \varphi}+\operatorname{sen} \theta \cos \varphi \frac{\partial}{\partial \theta}\right),
$$

Annali di Matematica, Serie III, tomo VIII. 
dove $k$ è una funzione da determinarsi. Ma, poichè

$$
\begin{aligned}
& \frac{\partial}{\partial \varphi}=-\operatorname{sen} \theta \operatorname{sen} \varphi \frac{\partial}{\partial e_{1}}+\operatorname{sen} \theta \cos \varphi \frac{\partial}{\partial e_{2}} \\
& \frac{\partial}{\partial \theta}=-\operatorname{sen} \theta \frac{\partial}{\partial e_{3}}+\cos \theta \operatorname{sen} \varphi \frac{\partial}{\partial e_{2}}+\cos \theta \cos \varphi \frac{\partial}{\partial e_{1}}
\end{aligned}
$$

si trova senz'altro

e quindi

$$
k=-\frac{1}{\operatorname{sen} \theta}
$$

$$
X_{2}=\operatorname{sen} \varphi \operatorname{cotg} \theta \frac{\partial}{\partial \varphi}-\cos \varphi \frac{\partial}{\partial \theta} .
$$

Analogamente :

$$
X_{1}=\operatorname{sen} \varphi \frac{\partial}{\partial \theta}+\cos \varphi \operatorname{cotg} \theta \frac{\partial}{\partial \theta} .
$$

Ampliamo ora il gruppo $X_{1}, X_{2}, X_{3}$ immaginandolo operante anche su $\sigma^{(1)}=\frac{d \theta}{d s}, \varphi^{(1)}=\frac{\partial \varphi}{\partial s}$. Avremo:

$$
\begin{gathered}
X_{3}=\frac{\partial}{\partial \varphi} ; \quad X_{2}=\operatorname{sen} \varphi \operatorname{cotg} \theta \frac{\partial}{\partial \varphi}- \\
+\cos \varphi \frac{\partial}{\partial \theta}+\varphi^{(1)} \operatorname{sen} \varphi \frac{\partial}{\partial \theta^{(1)}}+ \\
+\left[\varphi^{(1)} \cos \varphi \operatorname{cotg} \theta-\theta^{(1)} \frac{\operatorname{sen} \varphi}{\operatorname{sen}^{2}()}\right] \frac{\partial}{\partial \varphi^{(1)}} \\
X_{1}=\left(X_{3} X_{2}\right) .
\end{gathered}
$$

Lasciando ora il termine in $\frac{\partial}{\partial \varphi^{(1)}}$, perchè noi vogliamo soltanto occuparci di come il gruppo trasforma $\epsilon^{(3)}$, e osservando che $d s^{2}=d \theta^{2}+\operatorname{sen}^{2} \theta d c^{2}$, donde $\phi^{(1)}=\frac{\sqrt{1-\theta^{1 / 2}}}{\operatorname{sen} \theta}$, sostituendo alla $\zeta^{(1)}$ la nuova variabile $\psi=\int \frac{d \theta^{(1)}}{\sqrt{1-\theta^{(1) 2}}}$ il gruppo assume l'aspetto

$$
X_{3}=\frac{\partial}{\partial \varphi} \quad X_{2}=\operatorname{sen} \varphi \operatorname{cotg} \theta \frac{\partial}{\partial \varphi}-\cos \varphi \frac{\partial}{\partial \theta}+\frac{\operatorname{sen} \varphi}{\operatorname{sen} \theta} \frac{\partial}{\partial \psi}
$$

Ponendo dunque

$$
\begin{gathered}
X_{1}=\left(X_{3} X_{9}\right) . \\
x_{z}=\varphi \\
x_{1}=\theta \\
x_{3}=\psi
\end{gathered}
$$

questo gruppo assume proprio la forma data dal prof. Binnchi. 
Abbiamo dunque:

Le formule precedenti stabiliscono una rappresentazione del nostro spazio sulla sfera euclidea pensata come luogo di elementi, tale che ai movimenti dello spazio in sè corrispondono movimenti della sfera in sè stessa.

Se noi vogliamo che la rappresentazione sia biunivoca su tutta la sfera senza eccezione, resta senz'altro fissata la corrispondenza tra $\mathrm{i}$ punti dello spazio e le loro coordinate. E possiamo dire:

I gruppi finiti discontinui degli spazii in discorso sono quelli, che, nella corrispondenza precedentemente fissata, corrispondono ai gruppi dei poliedri regolari.

Il fatto che qui vediamo, cioè che $i$ gruppi finiti discontinui dei nostri spazii sono oloedricamente isomorfi a gruppi finiti discontinui di movimenti di uno spazio ellittico è un fatto generale, che si ripete sempre appena il gruppo totale dei movimenti non contenga trasformazioni infinitesime eccezionali. Infatti a un gruppo finito discontinuo di movimenti corrisponde un gruppo finito discontinuo nel gruppo aggiunto, che è in tal caso oloedricamente isomorfo al gruppo primitivo. E questo gruppo, essendo un gruppo finito di operazioni lineari lascia sempre invariata una forma quadratica definita che è p. es. la somma della forma $e_{1}^{2}+e_{2}^{2}+e_{3}^{2}$ e delle sue trasformate. Nel caso precedente questa forma è proprio “ $e_{1}^{2}+e_{2}^{2}+e_{3}^{2} n$.

Da queste osservazioni scaturisce senz'altro un metodo per ricercare in ogni caso cosiffatto se esistono nel gruppo considerato sottogruppi finiti discontinui. Il nostro metodo consiste nel formare il gruppo aggitnto, e di determinare quali delle sue trasformazioni lascino fissa una forma quadratica definita, cid che si compie con operazioni algebriche: considerata poi questa forina quadratica posta uguale a zero come assoluto di uno spazio ellittico, vedere quali sottogruppi discontinui finiti di questo spazio appartengono pure al gruppo aggiunto. In conclusione anche nel caso più generale la ricerca si riduce alla determinazione dei gruppi finiti discontinui di uno spazio ellittico. Applicheremo ora questo procedimento, un po'modificato, al tipo VIII ${ }^{\circ}$ del prof. Bianchi. Comincieremo dal costruirne il gruppo aggiunto. Esso viene definito dalle

$$
\begin{aligned}
& \frac{d e_{1}^{\prime}}{d t}+\lambda_{1} e_{2}^{\prime}-\lambda_{2} e_{1}^{\prime}=0 \\
& \frac{d e_{3}^{\prime}}{d t}+2 \lambda_{1} e_{3}^{\prime}-2 \lambda_{3} e_{1}^{\prime}=0 \\
& \frac{d e_{3}^{3}}{d t}+\lambda_{2} e_{3}^{\prime}-\lambda_{3} e_{2}^{\prime}=0 .
\end{aligned}
$$


Vediamo se qualche sua trasformazione lascia fissa una conica. E, poichè per le precedenti equazioni,

$$
e_{1}^{\prime} \frac{d e_{3}^{\prime}}{d t}+e_{3}^{\prime} \frac{d e_{1}}{d t}-\frac{1}{2} e^{\prime}{ }_{2} \frac{d e_{2}^{\prime}}{d t}=0
$$

ossia

$$
4 e^{\prime}{ }_{1} e_{3}-e_{2}^{\prime 2}=\text { cost. }
$$

tutte le trasformazioni del gruppo lasciano fissa la conica reale

$$
4 e^{\prime}{ }_{1} e^{\prime}{ }_{3}-e_{2}^{\prime 2}=0 \text {. }
$$

Allora senz'altro deduciamo che il nostro gruppo è oloedricamente isomorfo ai movimenti di una pseudosfera in sè perchè questa conica è reale, e, analogamente a quanto s'è fatto prima noi avremmo:

Il nostro spazio si pud riferire binnivocamente alla pseudosfera pensata come luogo dei suoi $\infty^{3}$ elementi in modo che ai movimenti della pseudosfera in sè stessa corrispondano movimenti dello spazio in sè. Questa corrispondenza definisce per noi senz'altio la corrispondenza tra un punto di $S_{3}$ e le sue coordinate. $\mathrm{E}$ allora senz'altro poichè eccetto casi banali, la pseudosfera non ammette gruppi finiti di movimenti in sè, ma ammette bensi gruppi infiniti discontinui, avremo:

Gli spazii in discorso non ammetlono (tranne qualche caso banale) gruppi funiti discontinui di movimenti: essi ammettono però infiniti gruppi infiniti discontinui di movimenti, isomorfi oloedricamente ai gruppi di Poincart.

Per gli altri tipi di $S_{3}$, che ammettono un $G_{3}$ transitivo di movimenti ò ben facile riconoscere l'impossibilità di gruppi discontinui finiti. Basta infatti riconoscere l'aperiodicità di una trasformazione qualsiasi del gruppo aggiunto. P. es, nel tipo $\mathrm{VI}^{\circ}$ il gruppo aggiunto è definito da

$$
\begin{aligned}
& \frac{d e_{1}^{\prime}}{d t}+\lambda_{1} e_{3}^{\prime}-\lambda_{3} e_{1}^{\prime}=0 \\
& \frac{d e_{3}^{\prime}}{d t}+h\left(\lambda_{2} e_{3}^{\prime}-\lambda_{3} e_{2}^{\prime}\right)=0 \\
& \frac{d e_{3}^{\prime}}{d t}=0 .
\end{aligned}
$$

donde, se $\lambda_{3}==_{1}^{\prime}=0$,

$$
\begin{aligned}
& e^{\prime}=\frac{\lambda_{1}}{\lambda_{3}}\left(e_{3}-e_{3} e^{\lambda_{3} t}\right)+e_{1} e^{\lambda_{\mathrm{s}} t} \\
& e_{2}^{\prime}=\frac{\lambda_{2}}{\lambda_{3}} e_{3}\left(1-e^{h \lambda_{3} t}\right)+e_{2} e^{h \lambda_{2} t} \\
& e_{3}^{\prime}=e_{3}
\end{aligned}
$$


che non può essere periodica nel campo reale, poichè, essendo $\lambda_{3}=0$, è $e^{\lambda_{3} t}=i=1$.

Se invece $\lambda_{3}=0$, si avrà:

$$
\begin{aligned}
& e_{1}^{\prime}=-\lambda_{1} e_{3} t+e_{1} \\
& e_{2}^{\prime}=-h \lambda_{2} e_{3} t+e_{2} \\
& e_{3}^{\prime}=e_{3}
\end{aligned}
$$

pure aperiodica se

$$
\lambda_{2}=0, \quad 0 \quad \lambda_{1}=0
$$

La discussione è analoga per gli altri tipi.

Invece ora di studiare quegli $S_{3}$ che ammettono un $G_{4}$, noi osserveremo che questi $G_{4}$ contengono una trasformazione infinitesima eccezionale e di. remo poche parole sui gruppi $G_{r}$ che contengono $k$ trasformazioni $Y_{1} \ldots Y_{k}$ infinitesime eccezionali. Per trovarne i sottogruppi finiti discontinui si trovino prima tutti i cosiffatti sottogruppi eventuali del gruppo aggiunto. Si determinano in un modo qualunque le trasformazioni corrispondenti del gruppo iniziale, cercando poi, se è possibile, di aggiungervi delle trasformazioni di quel sottogruppo di $G_{r}$ che viene generato dalle $Y_{1} \ldots Y_{k}$, che siano periodiche e che insieme alle precedenti generino effettivamente un gruppo della specie voluta.

$\S 13$. Daremo ora un'applicazione dei nostri metodi alla determinazione degli spazii che amrettono un $G_{1}$ oppure un $G_{2} \circ$ un $G_{3} \circ$ un $G_{4}$, oppure un gruppo a più di 4 parametri con sole 4 trasformazioni infinitesime indipendenti.

Per i primi quattro casi il problema si risolve facilmente: per il quinto caso invece si vedrà che occorrono nuovi e particolari artifici, senza i quali i calcoli diverrebbero estremamente lunghi e ben difficilmente condurrebbero in fondo.

A questo caso, ̀̀ riservata una prossima Memoria.

Per trovare gli spazii che ammettono un $G_{1}$ basta (cfr. Bianchi (A) pag. 8) supporre $\mathrm{i}$ coefficienti dell'elemento lineare indipendenti da $x_{1}$.

Per trovare gli spazii che ammettono un $G_{2}$, basta, nel primo caso, ammettere i coefficienti dell'elemento lineare indipendenti da $x_{1}, x_{2}$.

Nel secondo caso posto

$$
d s^{2}=\Sigma a_{i k} d x_{i} d x_{k} \quad(i=1,2, \ldots n)
$$


le formule di Killina ci danno subito che

$$
\begin{aligned}
& a_{2 k}=x_{1} a_{1 k}+r_{2 k} \quad(k==2) \\
& a_{22}=x_{1}^{2} a_{11}+2 c_{12} x_{1}+c_{22},
\end{aligned}
$$

dove le $c_{i k}$ sono arbitrarie, purchè non dipendano da $x_{1}, x_{2}$.

Spazii che ammettono un $G_{3}$ a trasformazioni infinitesime indipendenti.

Qui ricorderemo i risultati generali del $\S 4$. Per trovare gli spazii corrispondenti a uno di questi tipi noi dovremo scindere il calcolo in due parti: uno relativo alle $a_{i k}$ per $i, k=1,2,3$ e l'altro relativo alle $a_{i k}$ per $i>3, k \leq 3$. Quanto alle $a_{i k}$ dove $i>3, k>3$ noi sappiamo già che esse possono essere funzioni arbitrarie indipendenti dalle $x_{1}, x_{2}, x_{3}$. Esamineremo ora uno dopo l'altro i varii tipi di $G_{3}$, transitivi nelle $x_{1}, x_{2}, x_{3}$ già trovati.

1. tipo!. Per le $a_{i k}$ dove $i, k==1,2,3$ otteniamo, integrando le equazioni di Killing,

$$
\begin{aligned}
& a_{11}=c_{11} ; \quad a_{22}=c_{22} ; \quad a_{12}=e_{12} e^{\frac{x_{1}}{2}} ; \quad a_{23}=e^{x_{1}}\left(x_{1} c_{22}+c_{23}\right) \\
& a_{33}=e^{x_{1}}\left(x_{1}^{2} c_{22}+2 x_{1} c_{23}+c_{33}\right) \quad a_{13}=e^{\frac{x_{1}}{2}}\left(x_{1} c_{12}+c_{13}\right)
\end{aligned}
$$

dove le $c_{i k}$ sono indipendenti da $x_{1}, x_{2}, x_{3}$. Se lo spazio in discorso è un $S_{1}$, potremo supporre $a_{14}=1, a_{14}=a_{24}=a_{34}=0$ e le $c_{i k}$ funzioni di $x_{4}$. In generale arremo per le $a_{i k}$, dove $i>3, k \leq 3$

$$
\frac{\partial a_{i k}}{\partial x_{2}}=\frac{\partial a_{i k}}{\partial x_{3}}=0 ; \quad \frac{\partial a_{i 1}}{\partial x_{1}}=\frac{\partial a_{i 2}}{\partial x_{1}}-\frac{1}{2} a_{i 3}=\frac{\partial a_{i 3}}{\partial x_{1}}-a_{i 2}-\frac{1}{2} a_{i 3}=0
$$

equazioni, che si integrano senz'altro.

2. ${ }^{\circ}$ tipo). Per le $a_{i k}$ dove $i, k=1,2,3$ avremo per le equazioni di Krrlina, che subito si integrano,

$$
\begin{gathered}
a_{11}=c_{11} \quad a_{12}=c_{12} e^{x_{1}} \quad a_{13}=c_{13} e^{h x_{1}} \quad a_{22}=c_{22} e^{9 x_{1}} ; \quad a_{23}=c_{23} e^{(1+h) x_{1}} \\
a_{33}=c_{33} e^{2 h x_{1}}
\end{gathered}
$$

dove le $c_{i k}$ sono indipendenti da $x_{1}, x_{2}, x_{3}$.

Se lo spazio è un $S_{4}$ si può fare $a_{41}=1 \quad a_{i 4}=0(i=1,2,3)$.

In generale per $1, a_{i k}(i>3, k \leq 3)$ avremo:

$$
\frac{\partial a_{i k}}{\partial x_{2}}=\frac{\partial a_{i k}}{\partial x_{3}}=0 \quad-\frac{\partial a_{i k}}{\partial x_{1}}+a_{i 2} \frac{\partial x_{2}}{\partial x_{k}}+a_{i 3} h \frac{\partial x_{3}}{\partial x_{k}}=0
$$

che subito si integrano. 
3. ${ }^{\circ}$ tipo). Avremo per le $a_{i k}(i, k=1,2,3)$

$$
\begin{gathered}
\frac{\partial a_{i k}}{\partial x_{2}}=\frac{\partial a_{i k}}{\partial x_{3}}=0 ; \\
0=\frac{\partial a_{11}}{\partial x_{1}}=\frac{\partial a_{12}}{\partial x_{1}}+a_{13}=\frac{\partial a_{13}}{\partial x_{1}}-a_{12}+h a_{13}=\frac{\partial a_{22}}{\partial x_{1}}+2 a_{23}= \\
=\frac{\partial a_{23}}{\partial x_{1}}-a_{22}+h a_{23}+a_{33}=\frac{\partial a_{33}}{\partial x_{1}}-2 a_{23}+2 h a_{33} .
\end{gathered}
$$

Derivando la seconda di queste ultime equazioni rispetto a $x_{1}$ e portandovi il valore di $\frac{\partial a_{13}}{\partial x_{1}}$ dato dalla terza e tra la seconda e la terza formula cosl ottenuta eliminando $a_{13}$, si ottiene:

$$
\frac{\partial^{2} a_{12}}{\partial x_{1}^{2}}+h \frac{\partial a_{12}}{\partial x_{1}}+a_{12}=0 .
$$

$\mathrm{E}$, se $\alpha \pm i \beta$ sono le radici di $z^{2}+h z+1=0$ si avrà :

$$
a_{12}=\psi e^{\alpha x_{1}} \cos \beta x_{1}+\chi e^{\alpha x_{1}} \operatorname{sen} \beta x_{1}
$$

dove $\psi, \chi$ non dipendono da $x_{1}, x_{2}, x_{3}$.

Si ha quindi:

$$
a_{13}=-\frac{\partial a_{12}}{\partial c_{1}} \quad \frac{\partial^{2} a_{23}}{\partial x_{1}^{2}}-\frac{\partial a_{22}}{\partial x_{1}}+h \frac{\partial a_{23}}{\partial x_{1}}+\frac{\partial a_{33}}{\partial x_{1}}=0 .
$$

Sostituendo nell'ultima per le $\frac{\partial a_{22}}{\partial x_{1}}, \frac{\partial a_{23}}{\partial x_{1}}$ i loro valori, si ha:

$$
\frac{\partial^{2} a_{23}}{\partial x_{1}^{2}}+4 a_{23}+h \frac{\partial a_{23}}{\partial x_{1}}=2 h a_{33} \text {. }
$$

Derivando ed eliminando tra l'equazione così ottenuta e la precedente la $a_{33}$ si trova:

$$
\frac{\partial^{3} a_{23}}{\partial x_{1}^{3}}+3 h \frac{\partial^{2} a_{23}}{\partial x_{1}^{2}}+\left(4+2 h^{2}\right) \frac{\partial a_{23}}{\partial x_{1}}+4 h a_{23}=0 .
$$

Un'analoga equazione si troverebbe per $a_{22}$ e si arrebbe:

$$
\begin{aligned}
& a_{22}=\varphi e^{-h x_{1}}+\psi e^{-h x_{1}} \cos 2 \beta x_{1}+\chi e^{-h x_{1}} \operatorname{sen} 2 \beta x_{1} \\
& a_{23}=-\frac{1}{2} \frac{\partial a_{22}}{\partial x_{1}} \quad a_{33}=\frac{1}{2} \frac{\partial^{2} a_{22}}{\partial x_{1}^{2}}+\frac{\partial a_{22}}{\partial x_{1}}\left(1+\frac{h}{2}\right) .
\end{aligned}
$$


Si ha poi $a_{11}=\chi$, dove $\varphi, \psi, \chi$ sono funzioni indipendenti da $x_{1}, x_{2}, x_{3}$.

Se lo spazio è un $S_{4}$, potremo fare $a_{44}=1, a_{i 4}=0(i=1,2,3)$.

In generale per le $a_{i k}(i>3, k \leq 3)$ otteniamo:

$$
\frac{\partial a_{i k}}{\partial x_{2}}=\frac{\partial a_{i k}}{\partial x_{3}}=\frac{\partial a_{i k}}{\partial x_{1}}-a_{i 2} \frac{\partial x_{3}}{\partial x_{k}}+a_{i 3} \frac{\partial\left(x_{2}+h x_{3}\right)}{\partial x_{k}}=0
$$

ossia

$$
\frac{\partial a_{i 1}}{\partial x_{1}}=0 \quad \frac{\partial a_{i 2}}{\partial x_{1}}+a_{i 3}=0 \quad \frac{\partial a_{i 3}}{\partial x_{1}}+h a_{i 3}=0
$$

che si integrano senza difficoltà.

4..$^{\circ}$ tipo). Per le $a_{i k}(i, k=1,2,3)$ le equazioni e la loro integrazione si trova già nelle pag. 67-68 di (A). Basterà immaginarvi le $a, b, c, d$ funzioni indipendenti da $x_{1}, x_{2}, x_{3}$.

Per le $a_{i k}(i>3, k \leq 3)$ troviamo le:

$$
\begin{gathered}
\frac{\partial a_{i k}}{\partial x_{3}}=0 \quad \frac{\partial a_{i 1}}{\partial x_{1}}=\frac{\partial a_{i 2}}{\partial x_{2}}=\frac{\partial a_{i 3}}{\partial x_{2}}+a_{i 2}=0 \\
e^{-x_{3}} \frac{\partial a_{i k}}{\partial x_{1}}+a_{i 1} \frac{\partial\left(e^{\left.-x_{8}\right)}\right.}{\partial x_{k}}-a_{i 2} \frac{\partial\left(x_{2}^{2} e^{-x_{3}}\right)}{\partial x_{k}}-a_{i 3} \frac{\partial\left(2 x_{2} e^{-x_{n}}\right)}{\partial x_{k}}=0
\end{gathered}
$$

che si integrano senz'altro.

5..$^{\circ}$ tipo). Valgono per questo considerazioni perfettamente analoghe a quelle del tipo precedente.

6. ${ }^{\circ}$ tipo). Per le $a_{i k}(i, k=1,2,3)$ si trova, integrando le equazioni di Killina, e indicando con $\chi_{i k}$ delle funzioni indipendenti da $x_{1}, x_{2}, x_{3}$ che:

$$
\begin{gathered}
a_{11}=\chi_{11} ; \quad a_{12}=\chi_{12} ; \quad a_{13}=\chi_{13} ; \quad a_{13}=x_{1} \chi_{12}+\chi_{13} ; \quad a_{23}=x_{1} \chi_{22}+\chi_{23} \\
a_{33}=x_{1}^{2} \chi_{22}+2 x_{1} \chi_{23}+\chi_{33} .
\end{gathered}
$$

Per le $a_{i k}(i>3, k \leq 3)$ si ha:

$$
\frac{\partial a_{i k}}{\partial x_{2}}=\frac{\partial a_{i k}}{\partial x_{2}}=0 ; \quad \frac{\partial a_{i 1}}{\partial x_{1}}=\frac{\partial a_{i 2}}{\partial x_{1}}=\frac{\partial a_{i 3}}{\partial x_{1}}-\chi_{2}
$$

che tosto si integrano.

7. ${ }^{\circ}$ tipo). Basta supporre le $a_{i k}$ indipendenti da $x_{1}, x_{2}, x_{3}, x_{4}$.

Spazii che ammettono un $G_{3}$ a trasformazioni infinitesime linearmente dipendenti. In questo caso si dovrà scindere lo studio per le $a_{i k}$ in due altri casi, uno relativo alle $a_{i k}(i, k=2)$, l'altro per le $a_{i k}(i>2, k \leq 2)$. 
1. ${ }^{\circ}$ tipo). Tutte le $a_{i k}$ sono indipendenti da $x_{1}, x_{2}$. Per le $a_{i k}(i, k=1,2)$ si trova dalle formole di Killing che:

$$
a_{12}=a_{11}=c_{11} \quad a_{12}=0
$$

dove $c_{11}$ è indipendente da $x_{1}, x_{2}$.

Per le $a_{i k}(i>2, k \leq 2)$ si ha:

$$
\frac{\partial a_{i k}}{\partial x_{1}}=\frac{\partial a_{i k}}{\partial x_{2}}=0 \quad a_{i 1} \frac{\partial x_{2}}{\partial x_{k}}-a_{i 2} \frac{\partial x_{1}}{\partial x_{k}}=0
$$

che si integrano senz'altro.

2." tipo). Per le $a_{i k}(i, k=1,2$ ) si hanno le:

$$
\frac{\partial a_{11}}{\partial x_{1}}=0 \quad a_{12}=0 \quad \frac{\partial a_{22}}{\partial x_{1}}=2 a_{22} \operatorname{cotg} x_{1} \quad a_{22}=a_{11} \operatorname{sen}^{2} x_{1}
$$

che si integrano tosto.

Per le $a_{i k}(i>2, k \leq 2)$ le formule di Killing danno, poichè le $a_{t h}$ sono indipendenti da $x_{2}$, che:

$$
a_{i_{2}}=a_{i_{3}}=0 .
$$

$3 .^{\circ}$ tipo). La discussione è perfettamente analoga alla precedente.

Spazii che ammettono un gruppo $G_{4}$ a trasformazioni infinitesime dipendenti. La ricerca di questi spazii in generale si fa come precedentemente: noi, col solo fine di una maggiore brevità, studieremo soltanto gli $S_{4}$, accontentandoci di aver fatto rilevare dagli esempii precedenti il metodo generale.

1. ${ }^{\circ}$ tipo). Potremo chiaramente porre, quando ci si restringa agli $S_{4}$,

$$
d s^{2}=d x_{4}^{2}+\sum_{i, k} a_{i k} d x_{i} d x_{k} \quad(i, k=1,2,3) .
$$

Integrando le equazioni di KILLING, si trova

$$
a_{11}=c_{11} ; \quad a_{22}=c_{22} ; \quad a_{23}=x_{1} c_{22} ; \quad a_{33}=x_{1}^{2} c_{22}+c_{11} ; \quad a_{12}+a_{13}=0
$$

dove le $c_{i k}$ sono indipendenti da $x_{1}, x_{2}, x_{3}$.

2. ${ }^{\circ}$ tipo). Posto, come sopra

$$
d s^{2}=d x_{1}^{2}+\sum_{i, k} a_{i k} d x_{i} d x_{k} \quad(i, k=1,2,3)
$$

Annali di Matematica, Serie III, tomo VIII. 
otteniamo dalle equazioni di Killma,

$$
\begin{gathered}
a_{11}=c_{11}, \quad a_{33}=c_{33}, \quad a_{23}=n e^{x_{1}} c_{33}, \quad a_{12}=a_{13}=0, \\
a_{22}=e^{2 x_{1}}\left[\left(1-n^{2}\right) c_{11}+n^{2} c^{33}\right]
\end{gathered}
$$

dove ie $c_{i k}$ sono indipendenti da $x_{1}, x_{2}, x_{3}$.

3. ${ }^{\circ}$ tipo). Posto

$$
d s^{2}=d x_{i}^{2}+\sum_{i, k} a_{i k} d s_{i} d x_{k} \quad(i, k=1,2,3)
$$

si trova, con il solito procedimento, che :

$$
\begin{gathered}
a_{11}=c_{11} ; \quad a_{33}=c_{33} ; \quad a_{12}=a_{13}=0 ; \quad a_{23}=n c_{33} \cos x_{1} \\
a_{22}=n^{2} c_{33} \cos ^{2} x_{1}+c_{11} \operatorname{sen}^{2} x_{1} .
\end{gathered}
$$

Spazii che ammettono un gruppo $G_{4}$ non integrabile transitivo a trasformazioni infinitesime linearmente indipendenti.

Prima di trattare questo caso particolare, faremo alcune considerazioni generali sugli spazii che ammettono un $G_{4}$, a trasformazioni infinitesime indipendenti. Osserveremo cioè che in ognuno dei gruppi $G_{4}$ cosiffatti, precedentemente determinati, entrano degli elementi arbitrarii. E noi potremmo valercene per condurre in due maniere $i$ calcoli. Il primo modo consiste nel dare a questi elementi arbitrarii valori opportuni della massima semplicità, ricordando il teorema di Lre che due gruppi semplicemente transitivi sullo stesso numero di variabili e isomorfi sono anche simili. Il secondo metodo consisterebbe nel prefissare all'elemento lineare da determinarsi una forma saputa " a priori" possibile, lasciando quindi agli elementi arbitrarii che entrano nel gruppo tutta l'arbitrarietà compatibile con questa forma. Il primo metodo è più opportuno per lo studio degli spazii a un numero qualunque di dimensioni, il secondo per lo studio degli $S_{4}$. Diamo ora un esempio del primo metodo.

Prendiamo p. es. il gruppo $G_{4}$ generato dalle

$$
\begin{gathered}
X_{1}=\frac{\partial}{\partial x_{2}} ; \quad X_{2}=\frac{\partial}{\partial x_{3}} ; \quad X_{3}=-\frac{\partial}{\partial x_{1}}+x_{3} \frac{\partial}{\partial x_{2}} \\
X_{4}=\left[(\dot{c}-1) x_{1}+\varphi_{1}\right] \frac{\partial}{\partial x_{1}}+\left[-x_{1} \varphi_{3}+c x_{2}+\varphi_{2}\right] \frac{\partial}{\partial x_{2}}+ \\
+\left[x_{3}+\varphi_{3}\right] \frac{\partial}{\partial x_{3}}-\frac{1}{l_{4}} \frac{\partial}{\partial x_{4}} .
\end{gathered}
$$


Secondo i principii testè esposti potremo porre

$$
\varphi_{1}=\varphi_{2}=\varphi_{3}=0, \quad l_{4}=1
$$

col che si ha $X_{4}=(c-1) x_{1} \frac{\partial}{\partial x_{1}}+c x_{2} \frac{\partial}{\partial x_{2}}+x_{3} \frac{\partial}{\partial x_{3}}-\frac{\partial}{\partial x_{4}}$.

Per le $a_{i k}(i>4, k>4)$ basta supporre le funzioni indipendenti dalle $x_{1}, x_{2}, x_{3}, x_{4}$. Per le $a_{i k}(i, k=1,2,3,4)$ troviamo per le formule di Killina,

$$
\begin{gathered}
\frac{\partial a_{11}}{\partial x_{1}}=\frac{\partial a_{12}}{\partial x_{1}}=\frac{\partial a_{22}}{\partial x_{1}}=\frac{\partial a_{44}}{\partial x_{1}}=\frac{\partial a_{14}}{\partial x_{1}}=\frac{\partial a_{24}}{\partial x_{1}}=0 ; \\
\frac{\partial a_{33}}{\partial x_{1}}=2 a_{23} ; \quad \frac{\partial a_{i k}}{\partial x_{2}}=\frac{\partial a_{i k}}{\partial x_{3}}=0 \\
\frac{\partial a_{3}}{\partial x_{1}}=a_{i 2} \quad(1=1=3) \\
\frac{\partial a_{11}}{\partial x_{4}}=2(c-1) a_{11} ; \quad \frac{\partial a_{22}}{\partial x_{4}}=2 c ; \quad \frac{\partial a_{33}}{\partial x_{4}}=2 a_{33} ; \quad \frac{\partial a_{14}}{\partial x_{4}}=0 ; \\
\frac{\partial a_{12}}{\partial x_{4}}=(x-1) a_{12}+c a_{12}, \text { ecc., ecc. },
\end{gathered}
$$

che si integrano senz'altro. Per le $a_{i k}(i>4, k \leq 4)$ si hanno le:

$$
\begin{gathered}
\frac{\partial a_{i k}}{\partial x_{2}}=\frac{\partial a_{i k}}{\partial x_{3}}=0 ;-\frac{\partial a_{i k}}{\partial x_{1}}+a_{i 2} \frac{\partial x_{3}}{\partial x_{k}}=0 \\
(c-1) x_{1} \frac{\partial a_{i k}}{\partial x_{1}}-\frac{\partial a_{i k}}{\partial x_{4}}+(c-1) a_{i 1} \frac{\partial x_{1}}{\partial x_{k}}+c a_{i 2} \frac{\partial x_{2}}{\partial x_{k}}+a_{i 3} \frac{\partial x_{3}}{\partial x_{k}}=0
\end{gathered}
$$

che pure si integrano senza difficoltà. Con questo stesso metodo si possono chiaramente studiare tutti gli altri tipi di $G_{4}$. Noi, col solo scopo di non allungare questa Memoria con formule, che poi non utilizzeremmo, ci restringeremo agli $S_{4}$ e useremo naturalmente del secondo metodo. Cominciamo ora a trovare gli $S_{4}$ con un $G_{4}$ transitivo non integrabile del primo tipo, avvertendo che la prima parte dello studio ci servirà anche per $\mathrm{i}$ casi ulteriori. Siccome il sottogruppo formato dalle $X_{1}, X_{2}, X_{3}$ ha le $x_{4}=$ cost. come varietà invarianti potremo porre:

$$
d s^{2}=d x_{4}^{2}+\sum_{i: k} a_{i k} d x_{i} d x_{k} \quad(i, k=1,2,3) .
$$

Se $X f=\sum_{i=1}^{4} \xi_{i}\left(x_{k}\right) \frac{\partial f}{\partial x_{i}}$ è una trasformazione infinitesima che quello spa- 
zio ammette allora, scrivendo le condizioni di KıLLiva relative ad $a_{i k}=a_{41}$, $a_{42}, a_{43}, a_{44}$ otteniamo:

$$
\begin{gathered}
\partial \xi_{4}=0 \\
\partial x_{i}=0 \\
\sum_{k=1}^{3} a_{i k} \frac{\partial \xi_{k}}{\partial x_{4}}+\frac{\partial \xi_{4}}{\partial x_{i}}=0 \quad(i=1,2,3) .
\end{gathered}
$$

Indicando al solito con $A_{i k}$ il complemento algebrico di $a_{i k}$ diviso per il valore certamente non nullo del determinante $\left|a_{i k}\right|$ otterremo dalle $(\beta)$

$$
\frac{\partial \xi_{i}}{\partial x_{4}}=-\sum_{k=1}^{3} A_{i k} \frac{\partial \xi_{4}}{\partial x_{k}} \quad(i=1,2,3) \text {. }
$$

Nei casi in cui, come nel nostro, $\xi_{4}$ può essere soltanto funzione di $x_{4}$, esso ¿̀ quindi per la (a) costante, e perciò per le (I) abbiamo che: $\frac{\partial \xi_{1}}{\partial x_{4}}=\frac{\partial \xi_{2}}{\partial x_{4}}=\frac{\partial \xi_{3}}{\partial x_{4}}=0$. Dunque le $\zeta_{i}$ non possono contenere $x_{4} ;$ cosicchè la nostra scelta dell'elemento lineare $f a$ sì che tutte le funzioni arbitrarie (della $x_{4}$ ) che compariscono in $X_{4}$ sono semplici costanti. Queste considerazioni valgono anche per molti dei casi seguenti e noi non le ripeteremo.

Intanto avremo che le $\alpha, \beta, \gamma$ sono effettive costanti. $E$ se noi scriviano le formole di Kulung per le $a_{i k}(i, z=1,2,3)$ relative a $X_{1}, X_{2}, X_{3}$ otteniamo le formule pag. 67 di $\mathrm{B}$. $\mathrm{E}$ ci basterà dall'esame di $X_{4}$ ricavare le $a, b, c, d, e, f$ che nel nostro caso sono funzioni di $x_{4}$. Osserviamo intanto che nella $X_{4}$ potremo (dividendo le $\alpha, \beta, \gamma$ per una costante certo non nulla) supporre $l_{4}=1$. Scrivendo le equazioni di KuLLing, otteniamo dei polinomii in $x_{1}, x_{2}$ da uguagliarsi a zero. Annullando i coefficienti dei singoli termini (che saranno soltanto funzioni di $x_{4}$ ) raggiungeremo il nostro scopo. L'equazione di Killing relativa ad $a_{11}$ ci dà così:

$$
\frac{d\left(a^{2}\right)}{d x_{4}}=4\left(a^{2} \beta-\alpha b\right) \text {. }
$$

L'equazione di Kir.ung per $a_{33}$ ci dà, indicando con un apice la derivazione rispetto $x_{4}$ :

$$
\begin{gathered}
b^{\prime}=a^{2} \gamma+2 b \beta-\alpha(c+2 d) \\
c^{\prime}+2 d^{\prime}=6(b \gamma-e \alpha) \\
d^{\prime}=2(b \gamma-e \alpha) \\
e^{\prime}=(c+2 d) \gamma-2 e \beta-\alpha f .
\end{gathered}
$$


L'equazione di KıLLına relativa ad $a_{22}$ ci dà infine :

$$
f^{\prime}=4(e \gamma-f \beta) \text {. }
$$

Le $a, b, c, d, e, f$ si ottengono dunque integrando un sistema lineare di equazioni differenziali ordinarie del prim'ordine.

$2 .^{\circ}$ tipo). Prefisso come sopra all'elemento lineare la forma

$$
d x_{i}^{2}+\underset{i, k}{1,2,3} a_{i k} d x_{i} d x_{k}
$$

si trova che $\xi_{3}=\varphi, \xi_{4}=\psi$ sono costanti. Per le $a_{i k}(i, k=1,2,3)$ troviamo, poichè lo spazio deve ammettere il $G_{3} \equiv\left(X_{1}, X_{2}, X_{3}\right)$ le equazioni di pag. 69 (A) dove soltanto le costanti di integrazione saranno funzioni opportune di $x_{4}$. Se $\check{\xi}_{3}=0$, queste iunzioni saranno proprio costanti effettive; se $\xi_{3}=0$, notiamo che mutando $x_{4}$ in $K x_{4}$, dove $K$ è una costante opportuna, potremo fare $\xi_{3}=\xi_{4}$, mentre, sostituendo al nostro spazio uno spazio simile, potremo ancora porre $a_{44}=1$. Si ha cosi

$$
X_{4}=\frac{\partial}{\partial x_{3}}+\frac{\partial}{\partial x_{4}}
$$

e le $a_{i k}(i=1,2,3)$ si troveranno ancora date dalle formule a pag. 69 di (A), dove le $a, b, c \ldots$ si intendano ancora effettive costanti, ma al posto di $x_{3}$ si sostituisca $x_{3}-x_{4}$.

Spazii che ammettono un $G_{4}$ a trasformazioni linearmente indipendenti, integrabile e il cui gruppo derivato è un $G_{3}$ a trasformazioni infinitesime non permutabili.

1. tipo). Anche qui potremo porre

$$
d s^{2}=d x_{4}^{2}+\sum_{1, i} a_{i k} d x_{i} d \dot{x}_{k} \quad(i, k=1,2,3) .
$$

Avremo per l'osservazione fatta che $\varphi_{1}, \varphi_{2}, \varphi_{3}, l_{4}$ sono costanti effettive che indicheremo con $l_{1}, l_{2}, l_{3}, l_{4}$ cosicchè si potrà porre:

$$
\xi_{1}=(c-1) x_{1}+l_{1} \quad \xi_{2}=\varphi-x_{1} l_{3}+c x_{2} \quad \xi_{3}=x_{3} \quad \xi_{4}=-\frac{1}{l_{4}} .
$$

Essendovi le $X_{1}, X_{2}, X_{3}$ il nostro spazio sarà del tipo:

$$
\begin{gathered}
d s^{2}=d x_{4}^{2}+\varphi d x_{1}^{2}+2 \psi d x_{1} d x_{2}+\chi d x_{2}^{2}+2\left(x_{1} \psi+\delta\right) d x_{1} d x_{3}+ \\
+2\left(x_{1} \chi+\lambda\right) d x_{2} d x_{3}+\left(x_{1}^{2} \chi+2 x_{1} \lambda+\mu\right) d x_{3}^{2}
\end{gathered}
$$

dove le $\varphi, \psi, \chi, \delta, \lambda, \mu$ sono funzioni di $x_{4}$. 
Scrivendo le formule di Killiva relative a $X_{4}$ abbiamo:

$$
\begin{gathered}
{\left[(c-1) x_{1}+l_{1}\right] \frac{\partial a_{i k}}{\partial x_{1}}+a_{i_{1}}(c-1) \frac{\partial x_{1}}{\partial x_{k}}+a_{k_{1}}(c-1) \frac{\partial x_{1}}{\partial x_{i}}+} \\
+a_{i 2} \frac{\partial\left(c x_{2}-l_{3} x_{1}\right)}{\partial x_{k}}+a_{k 2} \frac{\partial\left(c x_{2}-l_{3} x_{1}\right)}{\partial x_{i}}+a_{3 i} \frac{\partial x_{3}}{\partial x_{k}}+a_{3 k} \frac{\partial x_{3}}{\partial x_{i}}=\frac{1}{l_{4}} \frac{\partial a_{i k}}{\partial x_{4}} .
\end{gathered}
$$

Sostituendovi i valori trovati delle $a_{i k}$, e integrando otteniamo:

$$
\begin{aligned}
& \chi=p_{22} e^{2 c l_{1} x_{4}} \\
& \psi=e^{(2 c-1) l_{1} x_{4}}\left\{-l_{3} p_{22} e^{l \cdot x_{4}}+p_{12}\right\} \\
& \varphi=e^{2 l .(c-1) x_{4}}\left\{-2 p_{12} l_{3} e^{l \cdot x_{4}}+p_{11}+l_{3}^{2} p_{22} e^{2 l_{3} x_{4}}\right\} \\
& \left.\lambda=e^{l_{4}(c+1) x_{4}}\left\{\frac{l_{1}}{c-1} p_{22} e^{l \cdot(c-1) x_{4}}+p_{23}\right\} \quad \text { (si noti che } c=_{1}^{\prime}=1\right) \\
& \mu=e^{2 l_{1} x_{4}}\left\{\frac{2 l_{1}}{c-1} p_{23} e^{l_{1}(c-1) x_{4}}+\frac{l_{1}^{2}}{(c-1)^{2}} p_{22} e^{2 l_{4}(c-1) x_{4}}+p_{33}\right\} \\
& \delta=e^{c l_{x_{4}}}\left\{\frac{l_{1} p_{12}}{c-1} e^{(c-1) l_{4} x_{4}}-l_{3} p_{23} e^{l_{1} x_{4}}-\frac{l_{1} l_{3}}{c-1} p_{22} \rho^{c l_{2} x_{4}}+p_{13}\right\}
\end{aligned}
$$

dove le $p_{i k}$ sono costanti effettive.

$2 .^{\circ}$ tipo). Anche qui si pone

$$
d s^{2}=d x_{4}^{2}+\sum_{i k} \alpha_{i k} d x_{i} d x_{k} .
$$

E se ne deduce che le $l_{i}$ sono effettive costanti. Per l'esistenza di $X_{1}$, $X_{2}, X_{3}$ si ha che $d s^{2}$ è del tipo già citato al $1 .^{\circ}$ caso.

Scrivendo le equazioni di Killing relative alla $X_{4}-l_{2} X_{1}-l_{3} X_{2}$ e integrando, troviamo:

$$
\begin{aligned}
& \chi=p_{22} e^{4 l_{1} x_{\downarrow}} \\
& \lambda=e^{3 l_{3} x_{4}}\left(p_{23}+l_{1} p_{22} e^{l_{3} x_{1}}\right) \\
& \mu=e^{2 l_{1} x_{4}}\left(l_{1}^{2} p_{22} e^{2 l_{2} x_{4}}+2 l_{1} p_{23} e^{t_{3} x_{4}}+p_{33}\right) \\
& \psi=e^{3 l_{1} x_{4}}\left\{-\left(l_{1}+l_{3}\right) p_{22} e^{l_{4} x_{4}}-l_{4} p_{23} x_{4}+p_{12}\right\} \\
& \delta=e^{2 l_{1} x_{1}} \mid p_{13}-l_{1}\left(l_{1}+l_{3}\right) p_{22} e^{2 l \cdot x_{4}}-\left(l_{1}+l_{3}\right) p_{23} e^{l_{1} x_{4}}+l_{1} p_{12} e^{l_{1} x_{4}}- \\
& -l_{4} p_{33} x_{4}-l_{1} l_{4} p_{23} x_{4} e^{l x_{4}} \\
& \varphi=e^{\circ} l_{x_{4}}\left\{\left(l_{1}+l_{3}\right)^{2} p_{22} e^{2 l_{1} x_{4}}-2\left(l_{1}+l_{3}\right) p_{12} e^{l \cdot x_{4}}+2 p_{23} l_{4} x_{4}\left(l_{1}+l_{3}\right) e^{l, x_{4}}+\right. \\
& \left.+l_{4}^{2} p_{33} x_{4}-2 l_{4} p_{13} x_{4}+p_{14}\right\}
\end{aligned}
$$

dove le $p_{i k}$ sono costanti. 
Spazii che ammettono un $G_{4}$ integrabile a trasformazioni infinitesime indipendenti, di cui il gruppo derivato è un $G_{2}$.

Per il primo dei due casi di questo tipo, troviamo col solito procedimento delle pagine precedenti e con notazioni analoghe:

$$
\begin{aligned}
d s^{2}=d x_{4}^{2}+\varphi d x_{1}^{2}+2 & \psi d x_{1} d x_{2}+\chi d x_{2}+2\left(x_{1} \psi+\cdot \vartheta\right) d x_{1} d x_{3}+ \\
& +2\left(x_{1} \chi+\lambda\right) d x_{2} d x_{3}+\left(x_{1}^{2} \chi+2 x_{1} \lambda+\mu\right) d x_{3}^{2}
\end{aligned}
$$

dove

$$
\begin{aligned}
& \chi=p_{22} e^{n l_{i} x_{4}} \\
& \psi=e^{l_{x_{4}}}\left(-l_{3} p_{22} e^{l^{. x_{1}}}+p_{12}\right) \\
& \varphi=-2 p_{12} l_{3} e^{l_{1} x_{4}}+l_{3}^{2} p_{22} e^{2 l_{4} x_{4}}+p_{11} \\
& \lambda=e^{0, x_{1}}\left(l_{1} l_{4} p_{22} x_{4}+p_{33}\right) \\
& \mu=e^{2 l_{1} x_{4}}\left\{2 l_{1} l_{4} p_{23} x_{1}+l_{1}^{2} l_{4}^{2} x_{1}^{2} p_{22}+p_{33}\right\} \\
& \approx=e^{l_{. x_{4}}}\left\{p_{13}+l_{1} l_{4} p_{12} x_{4}-l_{1} l_{3} p_{22} e^{l_{4} x_{4}}-l_{3} p_{23} e^{l_{4} x_{4}}+l_{1} l_{3} p_{22} e^{l_{4} \infty_{4}}-\right. \\
& -l_{1} l_{3} l_{4} p_{22} x_{4} e^{l_{4} x_{1}} ! \text {. }
\end{aligned}
$$

Studiamo i] secondo tipo di tali gruppi.

Esso contiene un $G_{3}$ a trasformazioni infinitesime indipendenti del tipo $2 .^{\circ}$, dove si faccia $h=0$.

Sarà perciò :

$$
\begin{aligned}
d s^{2}=d x_{4}^{2}+\psi d x_{1}^{2}+2 \psi e^{x_{1}} d x_{1} d x_{2} & +9 e^{2 x_{1}} d x_{2}^{2}+\mu d x_{3}^{2}+ \\
& +2 \chi d x_{1} d x_{3}+2 \lambda e^{x_{1}} d x_{2} d x_{3},
\end{aligned}
$$

dove le $\varphi, \psi, \ldots$ sono funzioni di $x_{4}$.

Nell'espressione trovata per la $X_{4} f$ saranno al solito le $\varphi_{i}$ vere costanti, e, come si vede percio tosto, potremo porre, indicando con $\alpha, \beta, l_{1}$ costanti,

$$
X_{4}=\alpha \frac{\partial}{\partial x_{1}}+\beta e^{-x_{1}} \frac{\partial}{\partial x_{2}}+x_{3} \frac{\partial}{\partial x_{3}}-\frac{1}{l_{4}} \frac{\partial}{\partial x_{4}} .
$$

Integrando le relative equazioni di Kinfing, e indicando con $p_{i k}$ delle costanti, abbiamo:

$$
\begin{aligned}
& \lambda=p_{23} e^{\left(x_{+1}\right) l_{x_{4}}} \\
& \mu=p_{33} e^{2 l_{2} x_{4}} \\
& \curvearrowright=p_{22} e^{2 \alpha l_{1} x_{+}}
\end{aligned}
$$




$$
\begin{aligned}
& \chi=e^{l, x_{1}}\left(-\frac{\beta}{\alpha} p_{23} e^{\alpha l_{1} x_{1}}+p_{13}\right) \\
& \psi=e^{\alpha l \cdot x_{4}}\left(-\frac{\beta}{\alpha} p_{22} e^{\alpha l_{1} x_{4}}+p_{12}\right) \\
& \varphi=-2 \beta\left(p_{12} \frac{1}{\alpha} e^{\alpha l_{1} x_{1}}-\frac{\beta}{2} p_{22}\left[\frac{e^{\alpha l_{4} x_{4}}}{\alpha}\right]^{2}+p_{11}\right) .
\end{aligned}
$$

Se $\alpha=0$, valgono le stesse formule, dove però al termine $\frac{e^{\alpha l_{3} x_{4}}}{\alpha}$ (che comparisce in $\varphi, \psi, \chi$ ) si deve sostituire il termine

$$
l_{4} x_{4}
$$

Spazii che ammettono un $G_{4}$ che contengono un $G_{3}$ a trasformazioni infinitesime permutabili e le cui trasformazioni infinitesime sono linearmente indipendenti.

In tutti i tipi già enumerati al $\S 11$ i coefficienti dell' elemento lineare corrispondente saranno funzioni della sola $x_{1}$. E noi potremo porre:

$$
d s^{2}=d x_{i}^{2}+\sum_{i, k} a_{i k} d x_{i} d x_{k} \quad(i, k=1,2,3) .
$$

Se ne ha che la $\varphi_{1}, \varphi_{2}, \varphi_{3}, l_{4}$ sono costanti.

Le equazioni di Kiluiva diventano:

$$
\begin{gathered}
2 \sum_{k=1}^{3} a_{i k} \frac{\partial \xi_{k}}{\partial x_{i}}+\xi_{4} \frac{\partial a_{i i}}{\partial x_{4}}=0 \quad(i=1,2,3) \\
\sum_{k=1}^{3} a_{i k} \frac{\partial \xi_{k}}{\partial x_{l}}+\sum_{l=1}^{3} a_{l k} \frac{\partial \xi_{k}}{\partial x_{i}}+\xi_{4} \frac{\partial a_{i l}}{\partial x_{4}}=0 \quad(i, l=1,2,3) .
\end{gathered}
$$

Sostituendovi per le $\xi_{i}$ i loro valori, integrando, indicando con $h_{i k}$ delle costanti, troviamo:

$\left.\mathrm{I}^{\circ}\right) a_{11}=h_{11} e^{2 l x_{2} x_{1}} \quad a_{22}=h_{22} e^{2 l_{i} \alpha x_{4}} \quad a_{33}==h_{33} e^{2 l_{1} x_{4}}$

$$
a_{12}=h_{12} e^{l_{1}(1+\alpha) x_{4}} \quad a_{23}=h_{23} e^{l_{2}(\alpha+c) x_{1}} \quad a_{13}=h_{13} e^{l_{1}(1+c) x_{4}}
$$

II $\left.^{0}\right) a_{12}=h_{12} e^{(1+2 c) l_{1} x_{\downarrow}} \quad a_{11}=h_{11} e^{2 l_{i} c x_{\downarrow}} \quad a_{22}=h_{22} e^{2 l_{1}(1+c) x_{\downarrow}}$

$$
\begin{aligned}
& a_{23}=\left(l_{4} h_{12} x_{4}+h_{23}\right) e^{l .(1+2 c) x_{4}} \quad a_{13}=e^{2 l_{2} c x_{4}}\left(l h_{11} x_{1}+h_{13}\right) \\
& a_{33}=e^{2 l_{1} c x_{4}}\left(l_{4}^{2} h_{11} x_{4}^{2}+2 h_{13} l_{4} x_{4}+h_{33}\right)
\end{aligned}
$$


$\left.\mathrm{III}^{\mathrm{o}}\right) a_{22}=h_{22} \quad a_{12}=l_{4} h_{22} x_{4}+h_{12} \quad a_{23}=\frac{1}{2} l_{1}^{3} h_{22} x_{4}+h_{12} l_{4} x_{4}+h_{z 3}$

$$
\begin{aligned}
& a_{13}=\frac{1}{2} l_{4}^{3} h_{22} x_{1}^{3}+\frac{3}{2} l_{4} h_{12} x_{4}^{3}+\left(h_{11}+h_{23}\right) l_{4} x_{1}+h_{13} \\
& a_{11}=l_{4} h_{22} x_{4}^{3}+2 h_{12} l_{4} x_{4}+h_{11} \\
& \quad a_{33}=\frac{l_{4}^{4}}{4} h_{22} x_{4}^{4}+l_{1}^{3} h_{12} x_{4}^{3}+l_{4}\left(h_{14}+h_{23}\right) x_{1}^{2}+2 l_{4} h_{33} x_{4}+h_{33}
\end{aligned}
$$

$\left.\mathrm{IV}^{\circ}\right) a_{22}=h_{22} e^{2 l x_{1}} ; \quad a_{12}=e^{2 l x_{1}}\left\{h_{22} l_{4} x_{4}+h_{12}\right\} ;$

$$
a_{11}=e^{2 l_{1} x_{4}}\left(l_{4}^{2} h_{22} x_{4}^{2}+2 l_{4} h_{12} x_{4}+h_{11}\right)
$$

$$
\begin{aligned}
& a_{23}=e^{2 l_{1} x_{4}}\left(l_{4}^{2} h_{22} \frac{x_{4}^{2}}{2}+l_{4} h_{12} x_{4}+h_{23}\right) ; \\
& a_{13}=e^{2 l_{4} x_{4}}\left\{h_{13}+\frac{l_{4}^{2} h_{22}}{2} x_{4}^{3}+\frac{3}{2} l_{4}^{2} h_{12} x_{4}^{7}+\left(h_{14}+h_{23}\right) l_{4} x_{4}\right\} \\
& a_{33}=e^{2 l_{1} x_{4}}\left\{h_{22} l_{4}^{4} \frac{x_{4}^{4}}{4}+l_{4}^{3} h_{12} x_{4}^{3}+\left(h_{11}+h_{23}\right) l_{4}^{2} x_{4}^{0}+2 l_{4} x_{4} h_{13}+h_{33}\right\}
\end{aligned}
$$

$\left.\mathrm{V}^{0}\right) a_{11}=h_{11} ; \quad a_{22}=h_{22} ; \quad a_{12}=h_{12} ; \quad a_{23}=l_{4} h_{22} x_{4}+h_{23} ;$

$$
a_{13}=l_{4} h_{12} x_{1}+h_{13}
$$

$a_{33}=l_{4}^{2} h_{22} x_{4}^{0}+2 l_{4} h_{33} x_{4}+h_{33}$

$\left.\mathrm{VI}^{0}\right) a_{11}=h_{11} e^{2 l_{1} x_{4}} \quad a_{22}=h_{22} e^{2 l_{3} x_{4}} \quad a_{12}=h_{12} e^{2 l_{3} x_{4}}$

$a_{13}=e^{2 l_{3} x_{4}}\left\{l_{4} h_{12} x_{1}+h_{13}\right\} \quad a_{23}=e^{2 l_{1} x_{4}}\left\{l_{4} h_{22} x_{4}+h_{23}\right\}$

$a_{33}=e^{2 l_{4} x_{4}}\left\{l_{4}^{2} h_{22} x_{4}^{2}+2 l_{4} h_{23} x_{4}+h_{33}\right\}$. 